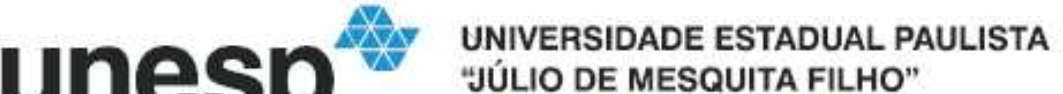 "JÚLIO DE MESQUITA FILHO" \\ Campus de Presidente Prudente
}

Programa de Pós-Graduação em Fisioterapia

Caracterização do perfil de atividade física na vida diária de pacientes portadores de DPOC do Brasil e comparação com pacientes da Áustria

Nidia Aparecida Hernandes Fujii

Presidente Prudente 


\section{Caracterização do perfil de atividade física na vida diária de pacientes portadores de DPOC do Brasil e comparação com pacientes da Áustria}

Nidia Aparecida Hernandes Fujii

Dissertação apresentada à Faculdade de Ciências e Tecnologia - FCT/Unesp para obtenção do Título de Mestre no Curso de Pós-graduação em Fisioterapia.

Orientador: Prof. Dr. Fábio Pitta

Presidente Prudente 


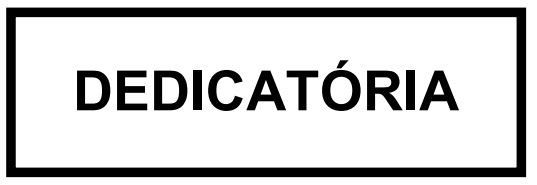


Ao meu esposo e aos meus pais que, em momento algum, hesitaram em me incentivar e apoiar minhas decisões.

Ao Prof. Antonio Fernando Brunetto (in memorian), exemplo de profissional e perseverança. 
AGRADECIMENTOS 
Ao meu orientador Prof. Dr. Fábio Pitta pela dedicação diária em construir minha formação científica, compartilhando seus conhecimentos e ensinando-me que os desafios me são impostos com um único objetivo... Superá-los. Por me ensinar que a honestidade vale a pena e que a confiança se constrói a cada dia.

Ao Prof. Antonio Fernando Brunetto (in memorian) pelo exemplo de perseverança. Com ele aprendi que enquanto há possibilidades, há chances de conquistar o que se deseja.

Aos professores Dra Ercy Mara Cipulo Ramos e Dr. Denilson de Castro Teixeira pela imensa contribuição ao constituir a banca de meu exame de Qualificação.

Aos professores Dra Dionei Ramos e Dr. Rubens Alexandre da Silva Junior por se disponibilizarem a contribuir com conhecimentos pedagógicos e científicos para o meu estudo.

Ao CNPq pelo apoio financeiro imprescindível para a realização dos presentes estudos.

Aos colegas do Laboratório de Pesquisa em Fisioterapia Pulmonar (LFIP) por colaborarem cientificamente com a execução dos estudos. Em especial, agradeço aos amigos Guto, Vinícius, Leandro, Mahara, Karina e Leila 
por proporcionarem tantos momentos de descontração e companheirismo e por acreditarem que um bom grupo de pesquisa cresce conjuntamente.

A todos os professores do Programa de Pós-graduação da FCT/Unesp por compartilharem seus conhecimentos e empenharem-se em construir um reconhecido curso de pós-graduação. 


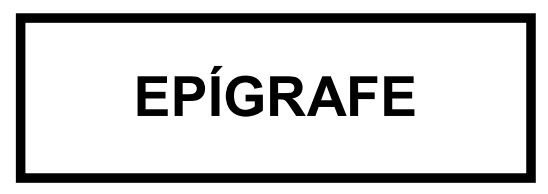


"A cada dia que vivo, mais me convenço de que o desperdício da vida está no amor que não damos, nas forças que não usamos, na prudência egoísta que nada arrisca e que, esquivando-nos do sofrimento, perdemos também a felicidade."

Carlos Drummond de Andrade 


\section{Apresentação \\ INTRODUÇÃO}

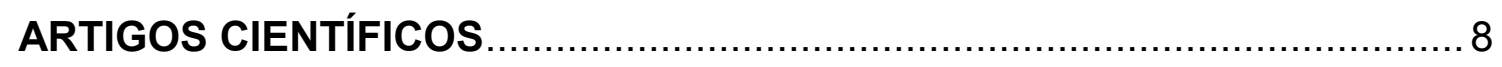

Artigo 1 "Perfil do nível de atividade física na vida diária de pacientes

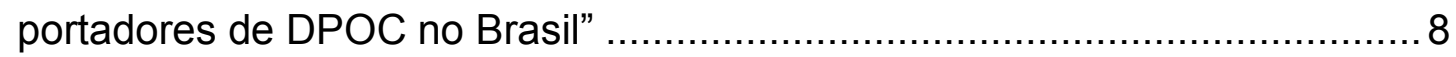

Artigo 2 "Comparison of daily physical activity level between stable COPD patients from Central-europe and South-america" ..................................... 28

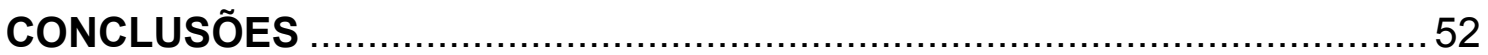

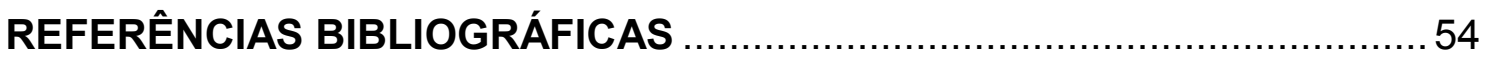

Anexos

Anexo 1 - Normas para publicação no periódico Jornal Brasileiro de

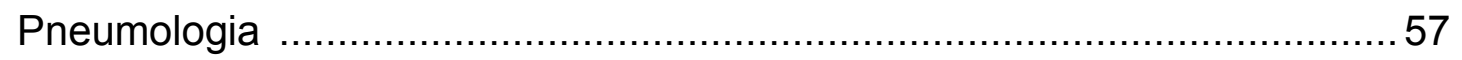

Anexo 2 - Normas para publicação no periódico Respiratory Medicine ........ 61 


\section{APRESENTAÇÃO}




\section{APRESENTAÇÃO}

Esta dissertação é composta de uma introdução e de dois artigos científicos, originados de pesquisas realizadas no Laboratório de Pesquisa em Fisioterapia Pulmonar do Departamento de Fisioterapia da Universidade Estadual de Londrina em conjunto com a Faculdade de Ciências e Tecnologia da Universidade Estadual Paulista Júlio de Mesquita Filho - Presidente Prudente. Em consonância com as regras do Programa de Pós-graduação em Fisioterapia desta instituição, os artigos foram redigidos de acordo com as normas dos periódicos Jornal Brasileiro de Pneumologia (anexo 1) e Respiratory Medicine (anexo 2).

Nidia A. Hernandes, Denilson C. Teixeira, Vanessa S. Probst, Antonio F. Brunetto, Ercy M. C. Ramos, Fabio Pitta. Perfil do nível de atividade física na vida diária de pacientes portadores de DPOC no Brasil. Publicado no Jornal Brasileiro de Pneumologia. 2009;35(10): 949-956.

pág. 08

Fabio Pitta, Marie-Kathrin Breyer, Nidia A. Hernandes, Denilson C. Teixeira, Thaís J. P. Sant'Anna, Andréa D. Fontana, Vanessa S. Probst, Antonio F. Brunetto, Martijn A. Spruit, Emiel F. M. Wouters, Otto C. Burghuber, Sylvia Hartl. Comparison of daily physical activity level between stable COPD patients from Central-Europe and South-America. Publicado na revista Respiratory Medicine. 2009; 103: 421-426.

pág. 28 
INTRODUÇÃO 


\section{INTRODUÇÃO}

Segundo o Global Initiative for Chronic Obstructive Lung Disease (GOLD) ${ }^{(1)}$, a Doença Pulmonar Obstrutiva Crônica (DPOC) é definida como: "uma doença prevenível e tratável com alguns efeitos extrapulmonares significantes que podem contribuir para a gravidade individualmente. 0 componente pulmonar da doença é caracterizado pela limitação ao fluxo aéreo que não é totalmente reversível. A limitação ao fluxo aéreo é geralmente progressiva e associada à resposta inflamatória anormal dos pulmões a partículas nocivas ou gases". Atualmente, a DPOC é a maior causa de morbidade e a quarta causa de mortalidade mundialmente ${ }^{(1)}$. Sua prevalência vem aumentando substancialmente devido ao envelhecimento da população e estima-se que, em 2020, a DPOC seja a terceira causa de morte mais comum no mundo ${ }^{(2)}$.

Além da dispnéia, tosse, sibilância, produção de secreção e infecções respiratórias de repetição, em muitos pacientes a doença está associada a diversas manifestações sistêmicas. Estas podem resultar efetivamente em piora da capacidade funcional, reduzindo a qualidade de vida e aumentando a mortalidade desses pacientes. As consequências sistêmicas incluem disfunção muscular esquelética, intolerância ao exercício, doença cardiovascular associada, perda de peso, desnutrição, ansiedade e depressão $(3 ; 4)$.

A disfunção muscular esquelética, uma das características extrapulmonares da doença, está ligada à diminuição da capacidade de 
exercício que, associada à dispnéia, levam à inatividade física. Isto configura o ciclo vicioso inatividade, descondicionamento e dispnéia que tem sido frequentemente mencionado na literatura ${ }^{(5)}$. A inatividade física tem repercussões importantes, sendo atualmente considerada como fator diretamente relacionado ao maior risco de hospitalização por exacerbação aguda e de mortalidade precoce em pacientes portadores de DPOC ${ }^{(6 ; 7)}$. O GOLD, referindo-se a esse "ciclo vicioso da DPOC", afirmou que "esses problemas possuem complexa relação e melhora em qualquer um desses processos interligados pode interromper o ciclo vicioso da DPOC e, então, ganhos positivos ocorrerão em todos os aspectos da doença" (1). Por esta razão, intervenções que possam aumentar o nível de atividade física desses pacientes apresentam potencial para romper tal ciclo vicioso e induzir à melhora importante. Por isso, é crescente o interesse em se avaliar acuradamente o nível de atividade física na vida diária (AFVD) em pacientes portadores de DPOC.

A AFVD pode ser quantificada por meio de observação direta, quantificação e/ou estimação do gasto energético, uso de questionários de atividade física auto-relatada e sensores de movimento. Observação direta e avaliação do gasto energético, apesar de acuradas, são métodos caros, complexos e de pouca aplicação clínica. Além disso, devido às importantes limitações no uso de questionários em populações inativas como os pacientes portadores de DPOC, a literatura recente recomenda que a avaliação objetiva do nível de AFVD seja realizada utilizando-se sensores de movimento ${ }^{(8)}$. 
Os sensores de movimento são equipamentos capazes de quantificar a atividade física realizada por um indivíduo num determinado período de tempo, bem como a intensidade da atividade. Desta forma, é possível avaliar detalhadamente as atividades de vida diária realizadas pelo paciente e determinar o seu perfil ${ }^{(8 ; 9)}$. Tal forma de avaliação objetiva também permite classificar os pacientes com sendo fisicamente ativos ou inativos, ou seja, se eles atingem o tempo mínimo diário de atividade física moderada recomendado pelo American College of Sports Medicine (por exemplo, 30 minutos caminhando por dia) ${ }^{(10)}$.

O Dynaport ${ }^{\circledR}$ Activity Monitor (McRoberts, Holanda) é um monitor de atividade física validado para pacientes portadores de DPOC e é considerado tão acurado quanto o método de observação direta para monitorização de AFVD ${ }^{(11)}$. Trata-se de um acelerômetro multiaxial capaz de detectar diferentes posturas e movimentos corporais. Este equipamento vem sendo amplamente utilizado em pesquisas clínicas ${ }^{(11-14)}$, pois é capaz de fornecer informações como o tempo gasto caminhando, em pé, sentado, deitado ou pedalando durante um período de tempo, bem como a intensidade de movimento durante a caminhada.

Alguns estudos prévios avaliaram em detalhes o nível de AFVD de pacientes portadores de DPOC europeus. Schönhofer e colaboradores (15) demonstraram que pacientes com DPOC da Alemanha apresentavam um menor nível de AFVD avaliado por meio de pedômetros quando comparados a indivíduos do grupo controle pareados para gênero e idade (3781 \pm 2320 passos/dia vs. $8590 \pm 4060$ passos/dia, $p<0,0001)$. Watz e colaboradores ${ }^{(16)}$ 
também estudaram pacientes portadores de DPOC alemães e concluíram que o nível de AFVD apresenta-se diminuído em pacientes a partir do GOLD II (grau de severidade moderado). Pitta e colaboradores ${ }^{(11)}$ observaram que pacientes portadores de DPOC da Bélgica apresentavam menor tempo caminhando por dia avaliado por meio de um monitor de atividade física quando comparados a idosos saudáveis $(44 \pm 26 \mathrm{~min} / \mathrm{dia} v \mathrm{~s} .81 \pm 26 \mathrm{~min} / \mathrm{dia}, \mathrm{p}$ $<0,0001)$.

A presença de pneumopatia crônica certamente não é o único fator a influir na redução da atividade física diária. Estudos realizados com populações de adultos e idosos saudáveis de diferentes países têm demonstrado que o nível de atividade física na vida diária pode ser influenciado por fatores ambientais, sócio-econômicos e culturais. Geralmente, um baixo nível de atividade física está associado à raça não-caucasiana, baixo grau de escolaridade e baixa renda financeira ${ }^{(17-20)}$. Entretanto, não se sabe ainda se tais afirmações podem ser aplicadas a populações sedentárias diagnosticadas com doenças crônicas, como a DPOC. Se o nível de AFVD for influenciado em pacientes portadores de DPOC da mesma forma que é influenciado em idosos e adultos saudáveis, é possível que pacientes do Brasil, um país multirracial, com baixo nível educacional e status social, sejam menos ativos em suas atividades diárias quando comparados a pacientes originários de um país com características opostas, como um país da Europa Central.

Em adição, considerando-se a hipótese de que características sociais, demográficas e antropométricas possam influenciar o nível de atividade física de pacientes com DPOC, torna-se necessário que se estude o perfil de 
AFVD de pacientes brasileiros, visto que, até o presente momento, apenas estudos com pacientes europeus foram publicados e seus resultados não podem, necessariamente, serem traduzidos para a população brasileira.

Por esta razão, os objetivos dos estudos presentes nesta dissertação foram os seguintes: 1) caracterizar o nível de atividade física na vida diária de uma amostra brasileira de pacientes portadores de DPOC e estudar as correlações entre variáveis fisiológicas (como função pulmonar e capacidade de exercício) e o nível de atividade física na vida diária nessa amostra; e 2) comparar as características do perfil de atividade física na vida diária entre pacientes portadores de DPOC de dois países com diferentes características étnicas e sócio-econômicas: Brasil e Áustria. 
ARTIGOS CIENTÍFICOS 


\section{ARTIGOS CIENTÍFICOS}

\section{Artigo 1}

Perfil do nível de atividade física na vida diária de pacientes portadores de DPOC no Brasil

Profile of physical activities in daily life in Brazilian patients with COPD

\section{Autores}

Nidia Aparecida Hernandes 1,3; Denilson de Castro Teixeira 1,2; Vanessa Suziane Probst 1,2; Antonio Fernando Brunetto 1; Ercy Mara Cipulo Ramos ${ }^{3}$; Fábio Pitta ${ }^{1,3}$.

\section{Instituições}

1 Laboratório de Pesquisa em Fisioterapia Pulmonar, Departamento de Fisioterapia, Universidade Estadual de Londrina, Londrina - PR.

${ }^{2}$ Centro de Ciências Biológicas e da Saúde, Universidade do Norte do Paraná, Londrina - PR.

3 Programa de Mestrado em Fisioterapia, Departamento de Fisioterapia, Faculdade de Ciências e Tecnologia, Universidade Estadual Paulista Júlio de Mesquita Filho, Presidente Prudente - SP.

\section{Endereço para correspondência}

Prof. Fábio Pitta

Departamento de Fisioterapia - Universidade Estadual de Londrina

Av. Robert Koch, 60 - Vila Operária - 86038-440, Londrina - PR, Brasil.

Telefone: (43) 33712477

E-mail: fabiopitta@uol.com.br

\section{Apoio financeiro}

Conselho Nacional de Desenvolvimento Científico e Tecnológico (CNPq) 


\section{RESUMO}

Objetivos: Avaliar as características de atividades físicas na vida diária de pacientes brasileiros portadores de DPOC e sua relação com diferentes variáveis fisiológicas. Métodos: Quarenta pacientes brasileiros portadores de DPOC (18 homens; $66 \pm 8$ anos; VEF $146 \pm 16 \%$ pred; IMC $27 \pm 6 \mathrm{Kg} \cdot \mathrm{m}^{-2}$ ) e 30 idosos saudáveis foram avaliados quanto às atividades físicas na vida diária utilizando-se um acelerômetro multiaxial (Dynaport ${ }^{\circledR}$ Activity Monitor, Holanda) por $12 \mathrm{~h} /$ dia durante 2 dias da semana. Foram ainda avaliados: capacidade máxima e funcional de exercício (teste incremental máximo e teste de caminhada de 6 minutos [TC6], respectivamente), pressões máximas inspiratória e expiratória (PImax e PEmax, respectivamente), força muscular periférica (1 repetição máxima e força de preensão manual), qualidade de vida (Saint George Respiratory Questionnaire [SGRQ]), estado funcional (questionário London Chest Activity of Daily Living [LCADL]) e sensação de dispnéia (escala do Medical Research Council [MRC]). Resultados: Pacientes portadores de DPOC apresentaram menor tempo gasto andando/dia quando comparados aos idosos saudáveis $(55 \pm 33$ versus $80 \pm 28 \mathrm{~min} /$ dia; $p=0,001)$ e menor intensidade de movimento $\left(1,9 \pm 0,4\right.$ versus $\left.2,3 \pm 0,6 \mathrm{~m} / \mathrm{s}^{2} ; \mathrm{p}=0,004\right)$. Os pacientes com DPOC também tenderam a passar mais tempo sentados $(294 \pm 114$ versus $246 \pm 122 \mathrm{~min} / \mathrm{dia} ; \mathrm{p}=0,08)$. O tempo andando/dia correlacionou-se com TC6 $(r=0,42 ; p=0,007)$, carga máxima de trabalho $(r=0,41 ; p=0,009)$, idade, MRC e domínio atividade do SGRQ $(-0,31 \leq r \leq-0,43$; $p \leq 0,05$ para todos). Conclusão: Apesar de serem mais ativos do que pacientes europeus estudados previamente, pacientes brasileiros portadores de DOPC são menos ativos em comparação a idosos saudáveis. O tempo gasto andando/dia é apenas moderadamente relacionado à capacidade máxima e funcional de exercício.

Palavras-chave: DPOC, atividade motora, tolerância ao exercício. 


\begin{abstract}
Objectives: To evaluate the characteristics of physical activities in daily life in Brazilian patients with Chronic Obstructive Pulmonary Disease (COPD) and the relationship of these characteristics with different physiologic variables. Methods: Forty Brazilian COPD patients (18 men; 66 \pm 8 years; FEV 1 $46 \pm 16 \%$ pred; BMI $27 \pm 6 \mathrm{Kg} \cdot \mathrm{m}^{-2}$ ) and 30 healthy age- and sex-matched subjects performed assessment of physical activity in daily life with an accelerometerbased activity monitor (Dynaport ${ }^{\circledR}$ Activity Monitor, The Netherlands) for 12 $\mathrm{h} /$ day in 2 weekdays. Other measurements included maximal and functional exercise capacity (incremental exercise test and six-minute walk test [6MWT], respectively), maximal inspiratory and expiratory pressures, peripheral muscle force (1-repetition maximum and handgrip force), quality of life (Saint George Respiratory Questionnaire, SGRQ), functional status (London Chest Activity of Daily Living questionnaire) and dyspnea sensation (Medical Research Council scale, MRC). Results: COPD patients had lower daily time spent walking in comparison to healthy elderly ( $55 \pm 33$ versus $80 \pm 28 \mathrm{~min} /$ day; $p=0.001)$ as well as reduced movement intensity $\left(1.9 \pm 0.4\right.$ versus $\left.2.3 \pm 0.6 \mathrm{~m} / \mathrm{s}^{2} ; \mathrm{p}=0.004\right)$. In addition, COPD patients also tended to have more daily sitting time $(294 \pm 114$ versus $246 \pm 122 \mathrm{~min} /$ day, $p=0.08)$. Time spent walking in daily life was correlated with 6MWT $(r=0.42 ; p=0.007)$, maximal workload $(r=0.41 ; p=0.009)$, age, MRC scale and $S G R Q$ activity domain $(-0.31 \leq r \leq-0.43$; all $p \leq 0.05)$. Conclusion: Despite being more active than previous reports of European COPD cohorts, Brazilian patients with COPD are less active in comparison to healthy elderly. Daily time spent walking in real life is only moderately related with maximal and functional exercise capacity.
\end{abstract}

Keywords: COPD, motor activity, exercise tolerance. 


\section{Introdução}

Além da obstrução ao fluxo aéreo, a Doença Pulmonar Obstrutiva Crônica (DPOC) é caracterizada por descondicionamento e inatividade física ${ }^{1-}$ 3. A disfunção muscular esquelética, uma das características extra-pulmonares da doença, está ligada à diminuição da capacidade de exercício que, associada à dispnéia, levam à inatividade física. A inatividade física na DPOC tem repercussões importantes, sendo hoje considerada como fator diretamente relacionado ao maior risco de exacerbações agudas e de mortalidade precoce ${ }^{4,5}$.

Atualmente, é crescente o interesse em se avaliar objetivamente o nível de atividade física na vida diária em diferentes populações, dentre elas os pacientes portadores de DPOC ${ }^{3,6}$. A literatura recente recomenda que, devido às importantes limitações no uso de questionários nessa população, a avaliação objetiva do nível de atividade física na vida diária seja realizada utilizando-se sensores de movimento, que são aparelhos portáteis que quantificam a atividade física realizada por um indivíduo num determinado período de tempo ${ }^{7,8}$. Essa avaliação objetiva permite, por exemplo, determinar se os pacientes atingem o nível mínimo diário de atividade física recomendado pelo American College of Sports Medicine (ACSM) para que um indivíduo seja considerado fisicamente ativo (30 minutos de atividade física moderada como caminhar) ${ }^{9}$.

Alguns estudos prévios avaliaram em detalhe as características da inatividade física de pacientes com DPOC em populações européias ${ }^{3,8, ~ 10-12 . ~}$ Dados recentes mostraram que pacientes brasileiros com DPOC apresentam um nível de atividade física maior do que uma população européia cuidadosamente pareada ${ }^{13}$. Porém, uma avaliação detalhada das características do nível de atividade física na vida diária em pacientes brasileiros com DPOC ainda não está disponível. Por exemplo, ainda não foi realizada uma comparação da atividade física diária entre pacientes brasileiros com DPOC e idosos saudáveis, assim como não se sabe qual proporção de pacientes brasileiros com DPOC atinge o nível mínimo de atividade física recomendado pelo American College of Sports Medicine.

O objetivo deste estudo foi caracterizar o nível de atividade física na vida diária de uma amostra brasileira de pacientes portadores de DPOC. 
Adicionalmente, objetivou-se estudar as correlações entre variáveis fisiológicas (como função pulmonar e capacidade de exercício) e o nível de atividade física na vida diária nessa amostra.

\section{Materiais e Métodos}

Quarenta pacientes brasileiros portadores de DPOC foram estudados (tabela 1). Todos foram recrutados durante a avaliação inicial para admissão no programa de Reabilitação Pulmonar do Hospital Universitário Regional do Norte do Paraná (HURNPr), Londrina, Paraná. O diagnóstico de DPOC foi estabelecido conforme os critérios do Global Initiative for Chronic Obstructive Lung Disease (GOLD) ${ }^{2}$. Além do diagnóstico de DPOC, os critérios de inclusão foram: estabilidade clínica, sem infecções e exacerbações nos últimos 3 meses; ausência de doença cardíaca grave e/ou instável; e ausência de alterações ósteo-neuro-musculares que limitassem as atividades físicas na vida diária. Dezessete (42\%) dos pacientes portadores de DPOC faziam uso de corticoesteróide via oral.

O grupo controle foi constituído por 30 indivíduos saudáveis e sedentários pareados aos pacientes com DPOC no que diz respeito à idade, gênero e características antropométricas. Os indivíduos do grupo controle foram recrutados dentre os parentes de alunos da Universidade Estadual de Londrina e membros da comunidade local. Este grupo tinha os mesmos critérios de inclusão do grupo com DPOC, com a ressalva de que deveriam apresentar espirometria normal e de que não deveriam ter histórico de realização de atividades físicas regulares no último ano.

Todos os pacientes portadores de DPOC eram aposentados e 13 deles $(32,5 \%)$ trabalhavam em atividades informais. Atividade informal foi considerada como qualquer atividade profissional ocasional e sem vínculo empregatício (poucos dias por semana, poucas horas por dia). No grupo controle, 27 indivíduos (90\%) eram aposentados e desses, 10 trabalhavam em atividades informais.

O projeto de pesquisa foi aprovado pelo Comitê de Ética em Pesquisa da Universidade Estadual de Londrina ( $\mathrm{n}^{\circ}$ 061/06). Todos os 
participantes receberam informações sobre os procedimentos realizados no estudo e assinaram um Termo de Consentimento Livre e Esclarecido.

A presença de comorbidades entre os indivíduos estudados está descrita na Tabela 2. Foram consideradas doenças cardíacas estáveis: miocardiopatia dilatada sem alteração significativa da função cardíaca, história de cardiopatia isquêmica, valvulopatia e arritmia atrial crônica. Presença de hipertensão arterial sistêmica foi considerada como comorbidade mediante necessidade de tratamento com anti-hipertensivos. Obesidade foi definida como índice de massa corpórea (IMC) acima de $30 \mathrm{~kg} / \mathrm{m}^{2}$. Osteoartrose, osteoporose, doenças da tireóide e desordem vascular foram relatadas pelos próprios participantes.

A atividade física na vida diária foi avaliada objetivamente por meio de um monitor de atividade física (Dynaport ${ }^{\circledR}$ Activity Monitor [DAM], McRoberts BV, Holanda). O aparelho consiste de uma pequena caixa posicionada na cintura e um sensor de atividade de membro inferior fixados ao indivíduo através de faixas (peso total de 375 gramas). O DynaPort registra o tempo gasto em diferentes atividades e posições corporais: andando, em pé, sentado ou deitado, bem como a intensidade de movimento medida durante o tempo em que o indivíduo está caminhando. $\mathrm{O}$ aparelho foi utilizado por 12 horas por dia durante dois dias de semana consecutivos, sendo utilizada a média dos dois dias para a análise estatística. O DynaPort foi validado em pacientes com DPOC ${ }^{14}$, e o número de dias mínimo necessário para obter-se uma avaliação confiável foi determinado em estudo prévio ${ }^{3}$. Após o uso do aparelho, um software específico (DynaScope, McRoberts BV, Holanda) foi utilizado para realizar a leitura e a análise dos dados coletados.

A prova de função pulmonar foi realizada em um espirômetro Pony (Cosmed, Itália) seguindo padronizações brasileiras ${ }^{1}$ e utilizando valores de referência de Pereira et al. ${ }^{16}$.

A capacidade funcional de exercício foi avaliada por meio do teste de caminhada de seis minutos (TC6), de acordo com padronização da American Thoracic Society ${ }^{17}$, sendo que os valores de referência foram aqueles descritos por Troosters et al. ${ }^{18}$. Para avaliação da capacidade máxima de exercício foi realizado o teste cardio-pulmonar de esforço (incremental máximo) de acordo com a padronização da American Thoracic 
Society/American College of Chest Physicians ${ }^{19}$ e seguindo protocolo previamente descrito ${ }^{20}$. O teste foi realizado em um cicloergômetro para membros inferiores (Monark, Suécia) com um analisador de gás portátil $\left(\mathrm{VO}_{2000}\right.$, Aeroport, Saint Paul, Minnesota, Estados Unidos) e um ecocardiograma digital (Micromed, Brasil).

A força muscular respiratória foi avaliada por meio da mensuração das pressões inspiratória e expiratória máximas (PImax e PEmax, respectivamente) conforme técnica descrita por Black e Hyatt ${ }^{21}$ e utilizando valores de referência descritos por Neder et al. ${ }^{22}$. Para avaliação da força muscular periférica foi realizado o teste de uma repetição máxima (1RM) utilizando um aparelho multi-estação de musculação (Riguetto, Brasil). Foram avaliados os grupos musculares: quadríceps femoral, bíceps e tríceps braquial. A força de preensão palmar foi avaliada por meio de um dinamômetro analógico (Jamar, Estados Unidos) e os valores de referência utilizados foram propostos por Mathiowetz et al. ${ }^{23}$.

O Saint George Respiratory Questionnaire (SGRQ), questionário específico para pacientes portadores de DPOC e validado em língua portuguesa ${ }^{24}$, foi aplicado para avaliação da qualidade de vida. O SGRQ é dividido em três domínios: sintomas, atividade e impacto psicossocial da doença. A pontuação é realizada em cada domínio, e um escore total é resultante da soma da pontuação dos três domínios. O estado funcional foi avaliado por meio da aplicação da escala London Chest Activity of Daily Living (LCADL), validada para a língua portuguesa ${ }^{25,26}$. A escala é dividida em quatro domínios: cuidado pessoal, cuidado doméstico, atividade física e lazer. É realizada pontuação em cada domínio e o escore total é resultante da soma de todos os domínios. A escala Medical Research Council (MRC), também validada em língua portuguesa ${ }^{27}$, foi utilizada para avaliação da sensação de dispnéia durante as atividades de vida diária. A escala é composta por apenas 5 itens, dentre os quais o paciente escolhe o item que corresponde a quanto a dispnéia limita sua vida diária.

$O$ índice BODE foi calculado a partir do índice de massa corpórea (IMC), volume expiratório forçado no primeiro segundo $\left(V_{E F}\right)$, sensação de dispnéia (escala MRC) e distância percorrida no TC6. A pontuação atribuída a cada item variou de zero a três para $\mathrm{VEF}_{1}, \mathrm{TC} 6$ e escala MRC, enquanto que 
ao IMC atribuiu-se zero ou um ponto. O escore total do índice BODE varia de zero a 10 pontos, e valores mais altos indicam maior risco de mortalidade.

Para a análise estatística foi utilizado o programa GraphPad Prism 3 (GraphPad Software, San Diego, Estados Unidos). Para avaliação da distribuição de normalidade dos dados foi utilizado o teste de KolmogorovSmirnov. Os dados foram descritos como média \pm desvio-padrão. O teste t não pareado foi utilizado para comparar o grupo de pacientes portadores de DPOC e o grupo controle. O coeficiente de Pearson foi utilizado para o estudo das correlações, com exceção daquelas envolvendo os escores do índice BODE, do questionário $S G R Q$ e das escalas LCADL e MRC, que foram avaliadas pelo coeficiente de Spearman. A significância estatística foi considerada como $\mathrm{p}<0,05$ para todas as análises.

\section{Resultados}

Os grupos foram pareados quanto à distribuição de gênero e não apresentaram diferença estatística quanto à idade e IMC. Além da obstrução ao fluxo aéreo e da redução da capacidade de exercício mostradas na tabela 1 , os portadores de DPOC apresentaram escore na escala MRC de $3 \pm 1$ pontos, índice BODE de $4 \pm 2$ e histórico de tabagismo de $38 \pm 35$ anos/maço. A tabela 1 mostra também que os idosos saudáveis apresentaram valores normais de função pulmonar e capacidade de exercício.

A Figura 1 mostra o tempo gasto por dia em diferentes atividades ou posições corporais em ambos os grupos. Pacientes portadores de DPOC passaram menos tempo andando por dia do que os idosos saudáveis $(55 \pm 33$ versus $80 \pm 28 \mathrm{~min} / \mathrm{dia} ; \mathrm{p}=0,001$ ), assim como sua intensidade de movimento da caminhada foi menor $\left(1,9 \pm 0,4\right.$ versus $\left.2,3 \pm 0,6 \mathrm{~m} / \mathrm{s}^{2} ; p=0,004\right)$. O grupo de pacientes portadores de DPOC tendeu também a passar mais tempo sentado

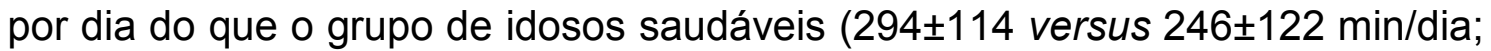
$\mathrm{p}=0,08)$. Os pacientes com DPOC passaram a maior parte do dia sentados ou deitados ( $41 \%$ e $15 \%$, respectivamente), enquanto o resto do tempo foi gasto locomovendo-se $(7 \%)$, em pé $(36 \%)$ ou em atividade/posição indefinida (1\%). Os idosos saudáveis passaram aproximadamente metade do dia locomovendose ou em pé ( $10 \%$ e $39 \%$, respectivamente), enquanto que o resto do tempo foi 
gasto na posição sentada (34\%), deitada (15\%) ou em atividade/posição indefinida (2\%).

Vinte e nove (72\%) pacientes portadores de DPOC passaram, em média, mais de 30 minutos por dia caminhando (Figura 2), sendo que $93 \%$ dos idosos saudáveis atingiram esse valor mínimo.

A Tabela 3 mostra que, em pacientes com DPOC, o tempo gasto andando por dia correlacionou-se positivamente com a distância percorrida no TC6 ( $r=0,42 ; p=0,007)$ (Figura 2), com a carga máxima de trabalho no teste cárdio-pulmonar de esforço $(r=0,41 ; p=0,009)$ e com o consumo máximo de oxigênio $\left(\mathrm{VO}_{2 \text { máx }}\right)(r=0,36 ; p=0,02)$. Além disso, o tempo gasto andando por dia correlacionou-se negativamente com idade, escala MRC e domínio atividade do SGRQ $(-0,31 \leq r \leq-0,43 ; p \leq 0,05$ para todos). O tempo gasto em pé por dia correlacionou-se positivamente com o volume expiratório forçado no primeiro segundo $\left(\mathrm{VEF}_{1}\right)$, relação $\mathrm{VEF}_{1} /$ capacidade vital forçada $(\mathrm{CVF})$ e ventilação voluntária máxima (VVM), em \% dos valores preditos $(0,41 \leq r \leq 0,43 ; p<0,05$ para todos) e negativamente com idade, relação anos/maço, índice BODE, domínio atividade do SGRQ e escore total do SGRQ $(-0,33 \leq r \leq-0,41 ; p<0,05$ para todos). A intensidade de movimento na vida diária correlacionou-se positivamente com o TC6 ( $r=0,64 ; p=0,0001)$, domínio sintomas do SGRQ $(r=0,37 ; p=0,02)$, carga máxima de trabalho no teste cardio-pulmonar de esforço $(r=0,62 ; p<0,0001)$ e $V_{2} O_{2 m a ́ x}(r=0,48 ; p=0,002)$, e negativamente com o domínio cuidados pessoais e escore total do LCADL, escala MRC, índice BODE e idade $(-0,32 \leq r \leq-0,58 ; p<0,05$ para todos). No grupo de idosos saudáveis apenas a intensidade de movimento correlacionou-se com a distância percorrida no TC6 $(r=0,49 ; p=0,005)$ e a idade $(r=-0,39 ; p=0,003)$.

\section{Discussão}

O presente estudo mostrou que pacientes portadores de DPOC brasileiros são menos ativos em suas atividades físicas de vida diária quando comparados a idosos saudáveis. Eles passaram a maior parte do tempo deitados ou sentados, além de caminharem com intensidade de movimento menor. Tais resultados podem ser atribuídos ao estilo de vida sedentário adotado por pacientes portadores de DPOC em conseqüência às alterações 
sistêmicas da doença, embora recentemente também tenha se levantado a hipótese contrária, ou seja, a inatividade pode ser precursora das alterações sistêmicas, e não sua consequência ${ }^{28}$.

Apesar de se mostrarem inativos fisicamente em relação a idosos saudáveis, os pacientes brasileiros com DPOC são mais ativos do que pacientes europeus pareados ${ }^{3,13}$. Isto possivelmente reflete o fato de que, em pacientes com DPOC, uma condição sócio-econômica inferior e uma maior miscigenação étnica parecem estar relacionadas a um maior nível de atividade física na vida diária, conforme previamente descrito ${ }^{13,29}$. Por outro lado, essa diferença não foi observada quando comparados os resultados dos idosos saudáveis do Brasil com o grupo de idosos saudáveis da Bélgica no estudo de Pitta et al. ${ }^{3}$. Tanto os idosos saudáveis brasileiros quanto os belgas passaram a maior parte do tempo locomovendo-se ou em pé, e a média de tempo gasto andando por dia foi similar entre eles $(80 \pm 28$ versus $81 \pm 26 \mathrm{~min} / \mathrm{dia}$, respectivamente). Isso sugere que os fatores determinantes do nível de atividade física em idosos saudáveis não são os mesmos que em pacientes com DPOC. Futuros estudos sobre esta questão são necessários.

Os resultados do presente estudo também revelaram correlação positiva entre tempo gasto andando por dia, distância percorrida no TC6 e carga máxima de trabalho no teste cárdio-pulmonar de esforço em pacientes com DPOC. A existência de correlação entre o nível de atividade física na vida diária e capacidade funcional de exercício já foi mostrada previamente, variando entre 0,46 e 0,74 em diferentes estudos ${ }^{3,6,30}$. No presente estudo, a correlação entre atividade física na vida diária e TC6 em pacientes brasileiros foi menor $(r=0,42)$, assim como a relação da atividade física na vida diária com a capacidade máxima de exercício $(r=0,41)$ foi menor do que em pacientes belgas $(r=0,64)^{2}$. Resultados prévios mostraram também que, em pacientes europeus, a distância percorrida no TC6 foi o principal fator determinante do tempo gasto andando por dia na vida diária em pacientes com DPOC $\left(r^{2}=0,56\right.$, $p<0.0001$ ) e os pacientes que caminharam menos de 400 metros no TC6 foram aqueles considerados extremamente inativos em sua vida diária ${ }^{3}$. Tal fato não foi observado em pacientes brasileiros, visto que não foi identificado um valor no TC6 que pudesse determinar qual indivíduo foi inativo ou não. O conjunto desses resultados indica uma relação menos estreita entre atividade física na 
vida diária e capacidade de exercício em pacientes brasileiros quando comparada a pacientes de outros países previamente estudados. Isso pode ser, pelo menos em parte, explicado pelo maior nível de atividade física em pacientes brasileiros em relação a pacientes desses outros países.

Baseado nas recomendações do American College of Sports Medicine ${ }^{9}$, a maioria dos pacientes com DPOC brasileiros $(72,5 \%)$ e dos idosos saudáveis brasileiros (93\%) podem ser considerados fisicamente ativos por atingirem o tempo mínimo andando recomendado por dia. Esse é um fato positivo, visto que Garcia-Aymerich et al. ${ }^{4}$ mostraram que indivíduos portadores de DPOC que realizam algum nível de atividade física regular apresentam menor mortalidade e menor risco de admissão hospitalar devido à doença. No entanto, tendo em vista que os pacientes brasileiros com DPOC se mostraram mais ativos em sua vida diária quando comparados a pacientes de populações européias previamente estudadas, é questionável se o mínimo de 30 minutos diários de caminhada sugerido pelo American College of Sports Medicine seja um valor aplicável para se qualificar indivíduos brasileiros como ativos ou não. Além disso, apesar da maioria dos pacientes com DPOC brasileiros serem considerados ativos, eles caminharam com intensidade de movimento $17 \%$ menor do que a dos idosos saudáveis $\left(2,0 \pm 0,4 \mathrm{~m} / \mathrm{s}^{2}\right.$ vs $2,3 \pm 0,6$ $\left.\mathrm{m} / \mathrm{s}^{2} ; \mathrm{p}=0,03\right)$, o que pode indicar que o benefício fisiológico obtido com a caminhada pode não ser o ideal.

As limitações do presente estudo incluíram o fato de a amostra pertencer a apenas uma localização do país (região sul do Brasil) e incluir indivíduos de uma faixa social relativamente homogênea, ou seja, indivíduos atendidos pelo Sistema Único de Saúde. Por outro lado, esses fatores podem não ter influenciado de forma significante os resultados, pois o clima da região de Londrina reflete bem o clima da maior parte do país (temperatura anual média de $20^{\circ} \mathrm{C}$, umidade relativa do ar de $78 \%$ e índice pluviométrico médio de $1400 \mathrm{~mm} / \mathrm{ano})^{3}$. Além disso, a renda mensal média dos indivíduos no presente estudo foi de $\mathrm{R} \$ 919,00$, refletindo a renda da grande maioria dos brasileiros. A ausência de pacientes portadores de DPOC leve (GOLD I) na presente amostra também pode ser considerada uma limitação, visto que não se pôde avaliar como se comporta esta parcela da população em relação aos idosos saudáveis. Outra limitação foi a quantidade de dias de monitorização da 
atividade física na vida diária (2 dias consecutivos, durante a semana). Estudo prévio mostrou que esse número de dias é suficiente para se obter uma estimativa válida em pacientes com DPOC utilizando-se o DynaPort activity monitor ${ }^{3}$, porém não há estudo prévio indicando o número de dias necessário para avaliar idosos saudáveis com esse aparelho. No entanto, acreditou-se que, por se tratar de indivíduos sedentários e aposentados, não haveria grande variabilidade no nível de atividade física entre os dias da semana. Além disso, não foi incluído o final de semana no período de monitorização, o que contribui para a diminuição da variabilidade dia-a-dia e para uma estimativa realista do nível habitual de atividade física nessa população.

Em resumo, os pacientes portadores de DPOC brasileiros são menos ativos em suas atividades físicas de vida diária quando comparados a idosos saudáveis, além de caminharem com menor intensidade de movimento. O tempo gasto andando na vida diária correlaciona-se apenas moderadamente com a capacidade funcional e máxima de exercício.

\section{Agradecimentos}

Os autores gostariam de agradecer a colaboração dos acadêmicos de Fisioterapia Renato Vitorasso e Mônica M. Guimarães e das fisioterapeutas Thaís J. P. Sant'Anna e Andréa D. Fontana.

\section{Referências}

1. Oga T, Nishimura K, Tsukino M, Sato S, Hajiro T. Analysis of the factors related to mortality in chronic obstructive pulmonary disease: role of exercise capacity and health status. Am J Respir Crit Care Med. 2003; 167(4): 544549.

2. Rabe KF, Hurd S, Anzueto A, Barnes PJ, Buist SA, Calverley P et al. Global strategy for the diagnosis, management, and prevention of chronic obstructive pulmonary disease: GOLD executive summary. Am J Respir Crit Care Med. 2007; 176(6): 532-555.

3. Pitta F, Troosters T, Spruit MA, Probst VS, Decramer M, Gosselink R. Characteristics of physical activities in daily life in chronic obstructive pulmonary disease. Am J Respir Crit Care Med. 2005; 171(9): 972-977.

4. Garcia-Aymerich J, Lange $P$, Benet $M$, Schnohr $P$, Anto JM. Regular physical activity reduces hospital admission and mortality in chronic 
obstructive pulmonary disease: a population based cohort study. Thorax. 2006; 61(9): 772-778.

5. Yohannes AM, Baldwin RC, Connolly M. Mortality predictors in disabling chronic obstructive pulmonary disease in old age. Age Ageing. 2002; 31(2): 137-140.

6. Watz $\mathrm{H}$, Waschki B, Meyer $\mathrm{T}$, Magnussen $\mathrm{H}$. Physical activity in patients with chronic obstructive pulmonary disease. Eur Respir J. 2009; 33(2): 262272.

7. Pitta F, Troosters T, Probst VS, Spruit MA, Decramer M, Gosselink R. Quantifying physical activity in daily life with questionnaires and motion sensors in COPD. Eur Respir J. 2006; 27(5): 1040-1055.

8. Pitta F, Troosters T, Probst VS, Lucas S, Decramer M, Gosselink R. Potential consequences for stable chronic obstructive pulmonary disease patients who do not get the recommended minimum daily amount of physical activity. J Bras Pneumol. 2006; 32(4):301-308.

9. Pate RR, Pratt M, Blair SN, Haskell WL, Macera CA, Bouchard C et al. Physical activity and public health. A recommendation from the Centers for Disease Control and Prevention and the American College of Sports Medicine. JAMA. 1995; 273(5): 402-407.

10. Coronado M, Janssens JP, Muralt B, Terrier P, Schutz Y, Fitting JW. Walking activity measured by accelerometry during respiratory rehabilitation. J Cardiopulm Rehabil. 2003; 23(5): 357-364.

11. Schonhofer B, Ardes P, Geibel M, Kohler D, Jones PW. Evaluation of a movement detector to measure daily activity in patients with chronic lung disease. Eur Respir J. 1997; 10(12): 2814-2819.

12. Singh S, Morgan MD. Activity monitors can detect brisk walking in patients with chronic obstructive pulmonary disease. J Cardiopulm Rehabil. 2001; 21(3): 143-148.

13. Pitta F, Breyer MK, Hernandes NA, Teixeira D, Sant'anna TJ, Fontana AD et al. Comparison of daily physical activity between COPD patients from Central Europe and South America. Respir Med. 2009; 103(3): 421-426.

14. Pitta F, Troosters T, Spruit MA, Decramer M, Gosselink R. Activity monitoring for assessment of physical activities in daily life in patients with chronic obstructive pulmonary disease. Arch Phys Med Rehabil. 2005; 86(10): 1979-1985.

15. Pereira CAC. I Consenso Brasileiro sobre Espirometria. J Bras Pneumol. 1996; 22(3): 105-158.

16. Pereira CAC, Barreto SP, Simões JG, Pereira FWL, Gerstler JG, Nakatani J. Valores de referência para espirometria em uma amostra da população brasileira adulta. J Bras Pneumol. 1992; 18(1): 10-22. 
17. ATS statement: guidelines for the six-minute walk test. Am J Respir Crit Care Med. 2002; 166(1): 111-117.

18. Troosters T, Gosselink R, Decramer M. Six minute walking distance in healthy elderly subjects. Eur Respir J. 1999; 14(2): 270-274.

19. ATS/ACCP Statement on cardiopulmonary exercise testing. Am J Respir Crit Care Med. 2003; 167(2): 211-277.

20. Probst VS, Troosters T, Pitta F, Decramer M, Gosselink R. Cardiopulmonary stress during exercise training in patients with COPD. Eur Respir J. 2006; 27(6): 1110-1118.

21. Black LF, Hyatt RE. Maximal respiratory pressures: normal values and relationship to age and sex. Am Rev Respir Dis. 1969; 99(5): 696-702.

22. Neder JA, Andreoni S, Lerario MC, Nery LE. Reference values for lung function tests. II. Maximal respiratory pressures and voluntary ventilation. Braz J Med Biol Res. 1999; 32(6): 719-727.

23. Mathiowetz V, Kashman N, Volland G, Weber K, Dowe M, Rogers S. Grip and pinch strength: normative data for adults. Arch Phys Med Rehabil. 1985; 66(2): 69-74.

24. Camelier A, Rosa FW, Salmi C, Nascimento OA, Cardoso F, Jardim JR. Avaliação da qualidade de vida pelo Questionário do Hospital Saint George na Doença Respiratória em portadores de doença pulmonar obstrutiva crônica: validação de uma nova versão para o Brasil. J Bras Pneumol. 2006; 32(2): 114-122.

25. Pitta F, Probst VS, Kovelis D, Segretti NO, Leoni A, Garrod R et al. Validation of the Portuguese version of the London Chest Activity of Daily Living Scale (LCADL) in chronic obstructive pulmonary disease patients. Rev Port Pneumol. 2008; 14(1): 27-47.

26. Carpes MF, Mayer AF, Simon KM, Jardim JR, Garrod R. The Brazilian Portuguese version of the London Chest Activity of Daily Living scale for use in patients with chronic obstructive pulmonary disease. J Bras Pneumol. 2008; 34(3): 143-151.

27. Kovelis D, Segretti NO, Probst VS, Lareau SC, Brunetto AF, Pitta F. Validation of the Modified Pulmonary Functional Status and Dyspnea Questionnaire and the Medical Research Council scale for use in Brazilian patients with chronic obstructive pulmonary disease. J Bras Pneumol. 2008; 34(12): 1008-1018.

28. Polkey MI, Rabe KF. Chicken or egg: physical activity in COPD revisited. Eur Respir J. 2009; 33: 227-229.

29. Garcia-Aymerich J, Felez MA, Escarrabill J, Marrades RM, Morera J, Elosua $\mathrm{R}$ et al. Physical activity and its determinants in severe chronic obstructive pulmonary disease. Med Sci Sports Exerc. 2004; 36(10): 1667-1673. 
30. Steele BG, Holt L, Belza B, Ferris S, Lakshminaryan S, Buchner DM. Quantitating physical activity in COPD using a triaxial accelerometer. Chest. 2000; 117(5): 1359-1367. 


\begin{tabular}{|c|c|c|}
\hline & $\begin{array}{l}\text { DPOC } \\
(n=40)\end{array}$ & $\begin{array}{c}\text { Idosos saudáveis } \\
(\mathrm{n}=30)\end{array}$ \\
\hline Idade (anos) & $66 \pm 8$ & $64 \pm 7$ \\
\hline Gênero $(H / M)$ & $18 / 22$ & $14 / 16$ \\
\hline $\operatorname{IMC}\left(\mathrm{Kg} / \mathrm{m}^{2}\right)$ & $27 \pm 6$ & $28 \pm 4$ \\
\hline GOLD (II/III/IV) & $17 / 17 / 6$ & - \\
\hline \multicolumn{3}{|l|}{ Função pulmonar } \\
\hline $\operatorname{VEF}_{1}$ (\% pred) & $46 \pm 16^{*}$ & $111 \pm 20$ \\
\hline CVF (\% pred) & $74 \pm 22^{*}$ & $109 \pm 20$ \\
\hline $\mathrm{VEF}_{1} / \mathrm{CVF}$ & $49 \pm 16^{*}$ & $82 \pm 5$ \\
\hline VVM (\% pred) & $41 \pm 19^{*}$ & $110 \pm 27$ \\
\hline \multicolumn{3}{|l|}{ Capacidade de exercício } \\
\hline TC6 $(\%$ pred $)$ & $72 \pm 18^{*}$ & $96 \pm 9$ \\
\hline TC6 (m) & $419 \pm 111^{*}$ & $560 \pm 75$ \\
\hline $\mathrm{W}_{\text {máx }}$ (watts) & $30 \pm 22$ & - \\
\hline $\mathrm{VO}_{2 \operatorname{máx}}\left(\mathrm{ml}^{\prime} \mathrm{Kg}^{-1} \cdot \mathrm{min}^{-1}\right)$ & $17,9 \pm 6,3$ & - \\
\hline
\end{tabular}

Tabela 1. Características dos indivíduos estudados.

DPOC: Doença Pulmonar Obstrutiva Crônica; IMC: índice de massa corpórea; GOLD: Global Initiative for Chronic Obstructive Lung Disease; $V_{E F}$ : volume expiratório forçado no primeiro segundo; CVF: capacidade vital forçada; VVM: ventilação voluntária máxima; TC6: teste de caminhada de seis minutos; $\mathrm{W}_{\text {máx: }}$ carga máxima de trabalho; $\mathrm{VO}_{2 m a ́ x}$ : consumo máximo de oxigênio.

Os valores foram descritos em média \pm desvio-padrão, com exceção de gênero e classe do GOLD.

Foi utilizado teste t não pareado para comparação entre os grupos.

${ }^{*} p<0,0001$ versus idosos saudáveis 


\begin{tabular}{|c|c|c|}
\hline Comorbidades & $\begin{array}{l}\text { DPOC } \\
(n=36)\end{array}$ & $\begin{array}{c}\text { Idosos saudáveis } \\
(n=30)\end{array}$ \\
\hline Obesidade* & $11(31 \%)$ & $11(37 \%)$ \\
\hline Doença cardíaca estável & $12(33 \%)$ & $10(33 \%)$ \\
\hline Hipertensão arterial sistêmica $^{\dagger}$ & $18(50 \%)$ & $17(57 \%)$ \\
\hline Diabetes & $13(36 \%)$ & $5(17 \%)$ \\
\hline Osteoartrose & $11(31 \%)$ & $10(33 \%)$ \\
\hline Osteoporose & $5(14 \%)$ & $4(13 \%)$ \\
\hline Doença da tireóide & $7(19 \%)$ & $3(10 \%)$ \\
\hline Doença vascular ${ }^{\ddagger}$ & $10(28 \%)$ & $11(37 \%)$ \\
\hline Tabagismo $^{\S}$ & $33(92 \%)$ & $9(30 \%)$ \\
\hline
\end{tabular}

Tabela 2. Presença de comorbidades nos pacientes portadores de Doença Pulmonar Obstrutiva Crônica (DPOC) e nos idosos saudáveis.

DPOC: Doença Pulmonar Obstrutiva Crônica. Não foi possível realizar o levantamento de comorbidades em 4 pacientes com DPOC.

* Índice de massa corpórea $>30 \mathrm{Kg} / \mathrm{m}^{2}$

${ }^{\dagger}$ Necessidade de tratamento com medicamento anti-hipertensivo

${ }^{\ddagger}$ Insuficiência arterial periférica ou trombose venosa

$\S$ Ex-tabagistas e tabagistas 


\begin{tabular}{|l|c|c|}
\hline & Tempo andando/dia & Tempo em pé/dia \\
\hline Idade & $-0,43^{* *}$ & $-0,33^{*}$ \\
\hline Anos/maço & $-0,10$ & $-0,34^{*}$ \\
\hline MRC & $-0,31^{*}$ & $-0,25$ \\
\hline BODE & $-0,30$ & $-0,34^{*}$ \\
\hline SGRQ atividade & $-0,33^{*}$ & $-0,41^{*}$ \\
\hline SGRQ total & $-0,27$ & $-0,41^{* *}$ \\
\hline Função pulmonar & & \\
\hline VEF $1 \%$ pred & 0,17 & $0,41^{* *}$ \\
\hline VEF 1 CVF & 0,02 & $0,41^{\text {** }}$ \\
\hline VVM \%pred & $-0,02$ & $0,43^{* *}$ \\
\hline Capacidade de exercício & & \\
\hline TC6 & $0,42^{* *}$ & 0,12 \\
\hline W $_{\text {máx }}$ & $0,50^{* *}$ & 0,19 \\
\hline VO $_{2 \text { máx }}$ & $0,36^{*}$ & 0,15 \\
\hline
\end{tabular}

Tabela 3. Correlações entre tempo gasto andando e em pé por dia durante diferentes atividades físicas na vida diária em pacientes brasileiros portadores de Doença Pulmonar Obstrutiva Crônica (DPOC).

MRC: Escala do Medical Research Council; SGRQ: Saint George Respiratory Questionnaire; $V_{E F}$ : volume expiratório forçado no primeiro segundo; VVM: ventilação voluntária máxima; TC6: teste de caminhada de seis minutos; $W_{\text {máx: }}$ : carga máxima de trabalho; $\mathrm{VO}_{2 m a ́ x}$ : consumo máximo de oxigênio. Foi utilizado o coeficiente de correlação de Pearson, com exceção das variáveis: BODE, $\mathrm{MRC}, \mathrm{SGRQ}$ e $\mathrm{VO}_{2 \text { máx }} \%$ predito, para as quais que foi utilizado o coeficiente de correlação de Spearman.

${ }^{*} p \leq 0,05$

** $p<0,01$ 


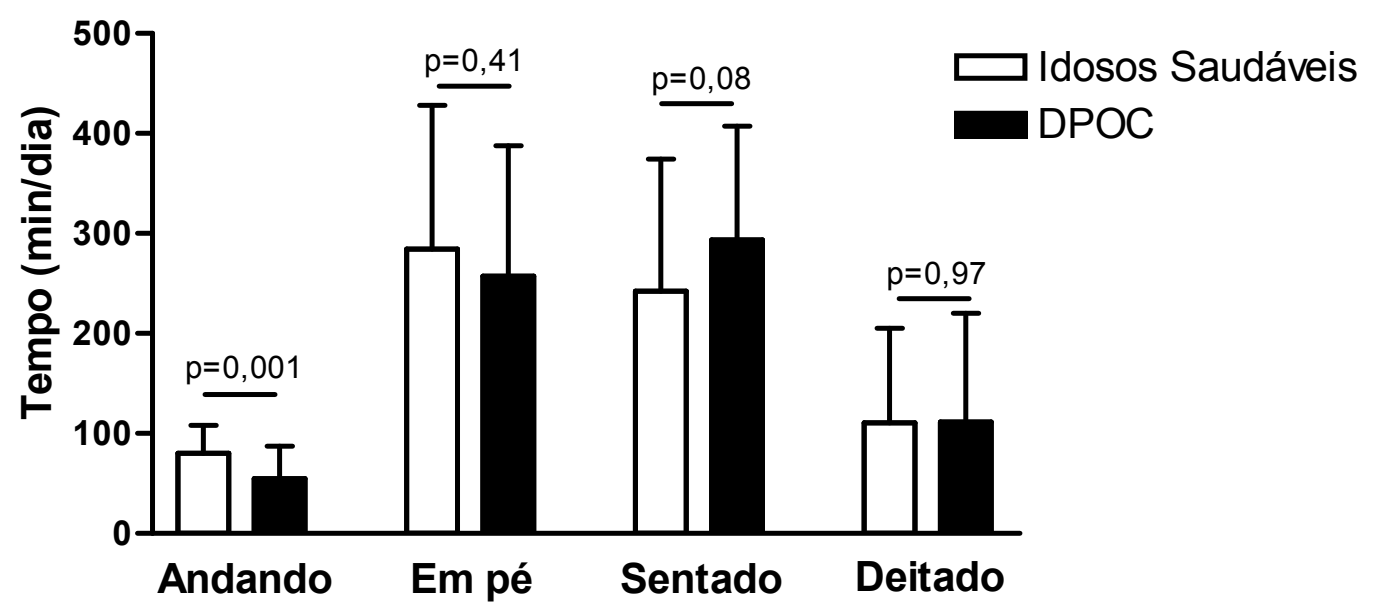

Figura 1. Tempo gasto por dia em diferentes atividades e posições corporais em pacientes portadores de Doença Pulmonar Obstrutiva Crônica (DPOC) e idosos saudáveis.

O teste t não pareado foi utilizado para a comparação entre os grupos. 


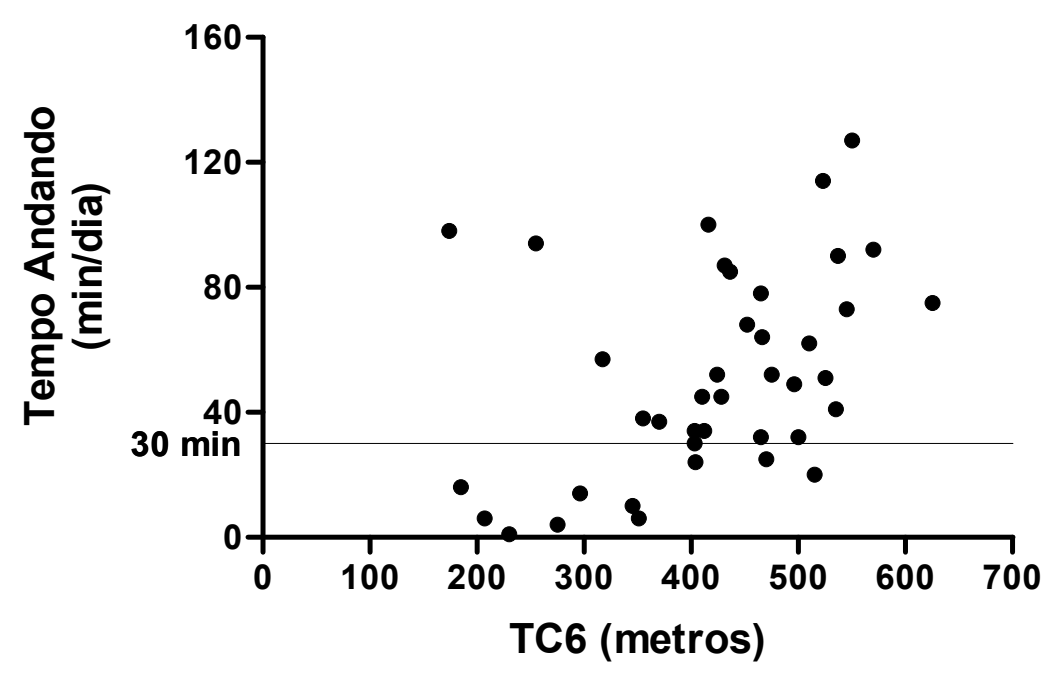

Figura 2. Correlação entre tempo andando por dia e o teste da caminhada de 6 minutos (TC6) em pacientes portadores de Doença Pulmonar Obstrutiva Crônica (DPOC) $(r=0,42 ; p=0,007)$.

A linha pontilhada posicionada em 30 minutos corresponde ao nível mínimo de atividade física diária recomendado pelo American College of Sports Medicine. 


\section{Artigo 2}

COMPARISON OF DAILY PHYSICAL ACTIVITY LEVEL BETWEEN STABLE COPD PATIENTS FROM CENTRAL-EUROPE AND SOUTH-AMERICA

Fábio Pitta ${ }^{1, *}$, Marie-Kathrin Breyer ${ }^{2,3}$ *, Nidia A. Hernandes ${ }^{1}$, Denilson Teixeira ${ }^{1}$, Thaís J. P. Sant'Anna ${ }^{1}$, Andréa D. Fontana ${ }^{1}$, Vanessa S. Probst ${ }^{1}$, Antonio F. Brunetto $^{1}$, Martijn A. Spruit ${ }^{3}$, Emiel F. M. Wouters ${ }^{3,4}$, Otto C. Burghuber ${ }^{2}$, Sylvia Hartl $^{2}$.

* Both authors contributed equally to the study

1 - Laboratório de Pesquisa em Fisioterapia Pulmonar (LFIP), Departamento de Fisioterapia, Universidade Estadual de Londrina (UEL), Londrina, Brazil.

2 - Department of Respiratory and Critical Care Medicine and Ludwig Boltzmann Institute for COPD, Otto Wagner Hospital, Vienna, Austria.

3 - Department of Research Development and Education, Centre for Integrated Rehabilitation of Organ failure (CIRO), Horn, The Netherlands.

4 - Respiratory Medicine, University Hospital Maastricht, Maastricht, The Netherlands

\section{Corresponding author:}

Prof. Dr. Fábio Pitta

Departamento de Fisioterapia - CCS - HURNPR

Rua Robert Koch, 60 - Vila Operária, 86038-440 - Londrina - PR - Brasil

Phone: +55 $4333712288 \quad$ Fax: +55 4333712459

E-mail: fabiopitta@uol.com.br

Short title: Inactivity in COPD patients from Austria and Brazil 


\section{ABSTRACT}

In healthy elderly and adults, lower physical activity level in daily life has been associated with lower socio-economic level and non-Caucasian race. The objective of this study was to test whether this is also applicable in Chronic Obstructive Pulmonary Disease (COPD) by comparing physical activity levels in daily life in stable patients from two countries with different socio-economic and ethnic realities: Austria and Brazil.

Physical activity in daily life was objectively assessed in 40 Austrian and 40 Brazilian COPD patients. Groups were matched for age, gender, body mass index, disease severity, smoking history, presence of concomitant heart disease, lung function, dyspnea and functional exercise capacity. In addition, climatic conditions were similar during the period of data collection in the two groups.

In comparison to Brazilian patients, Austrian patients had significantly lower walking time $(p=0.04)$, higher sitting time $(p=0.02)$ and lower movement intensity $(p=0.0001)$. The proportion of patients who did not reach an average of 30 minutes of walking per day was $48 \%$ in the Austrian group and $23 \%$ in the Brazilian group.

Austrian patients with COPD showed a significantly lower daily physical activity level in comparison to matched Brazilian patients. Socio-economic and ethnic factors appear to influence stable COPD patients differently than described in previous studies for healthy elderly and adults.

Keywords: activities of daily living; activity monitoring; Chronic Obstructive Pulmonary Disease; population comparison. 


\section{INTRODUCTION}

Objective and accurate assessment of physical activity level in daily life has been made more widely available due to recent technological advances. The use of real-life activity monitors has allowed to study in detail the characteristics of daily physical activity in various populations, including patients with Chronic Obstructive Pulmonary Disease (COPD) ${ }^{(1)}$. It has been shown, for example, that patients with COPD from Belgium were significantly less active than their age- and gender-matched healthy elderly compatriots ${ }^{(2)}$. Similar findings were found in studies performed in different European countries such as England ${ }^{(3)}$, Germany ${ }^{(4)}$ and Switzerland ${ }^{(5)}$. However, to the authors' knowledge, a comparison of time spent actively in daily life between COPD patients from different countries and continents using advanced activity monitors has never been performed. This may be of clinical interest since it allows investigating whether and to what extent COPD patients' profile of daily physical activity differs across diverse world regions.

In healthy elderly and adult populations, studies performed in different countries have shown that the amount of physical activity performed in daily life may vary considerably depending on various factors such as ethnicity, education level and social class ${ }^{(6-10)}$. In general, a lower physical activity level was associated with non-Caucasian race ${ }^{(6 ; 7)}$, lower educational level ${ }^{(8 ; 9)}$ and lower income ${ }^{(9 ; 10)}$. However, it is unknown whether these factors also have the similar influence on the physical activity level of a physically inactive population such as patients with COPD. In case they do, it is likely that patients from a country with a multi-racial population, lower educational level and social status 
(such as Brazil) will have lower physical activity level in comparison to a country with opposite characteristics (such as Austria).

The objective of the present study was to compare the level of physical activity in daily life of patients with COPD from Brazil with a matched group of patients with COPD from Austria.

\section{METHODS}

\section{Design}

In this cross-sectional study, the level of physical activity in daily life was assessed using an advanced activity monitor in a group of Brazilian patients with COPD and a group of Austrian patients with COPD for comparison. Austrian and Brazilian patients were matched for age, gender, body mass index (BMI), smoking history, presence of concomitant heart disease, lung function (spirometry), self-reported degree of dyspnea in daily life (modified version of the Medical Research Council scale - MMRC), functional exercise capacity (6minute walking test $-6 \mathrm{MWT}$ ) and disease severity as assessed by the GOLD stage and the BODE index. In addition, climatic conditions were similar during the period of data collection in the two groups since data in Brazil were collected during the whole year of 2006, whereas data collection in Austria occurred almost entirely during the Summer and Spring seasons of 2006 (see more details below).

\section{Subjects}

The study included 40 Brazilian patients with COPD (18 male; 66 \pm 8 years; forced expiratory volume in the first second $\left[\mathrm{FEV}_{1}\right] 46 \pm 17 \%$ predicted; 
BMI $26 \pm 6 \mathrm{~kg} / \mathrm{m}^{2}$ ) and 40 matched Austrian patients with COPD (21 male; $63 \pm 7$ years; $F E V_{1} 48 \pm 17 \%$ predicted; BMI $26 \pm 4 \mathrm{~kg} / \mathrm{m}^{2}$ ), all of them in initial screening for admission to a pulmonary rehabilitation program in their respective centers. The diagnosis of COPD was established based on internationally accepted criteria determined by the Global Initiative for Chronic Obstructive Lung Disease (GOLD) (11). No patient in both groups was using domiciliary long-term oxygen therapy (LTOT). They were all officially retired, although 5 patients in the Austrian group (12.5\%) and 14 patients in the Brazilian group (35\%) were still performing "informal" professional activities. By "informal" we considered no formal employment implied, i.e., occasional and not registered work (few hours per week, not every day). No patient in both groups took part in any rehabilitation program in the last year before entering the study. Inclusion criteria were: clinical stability (absence of exacerbations) for at least 3 months before inclusion in the study; and absence of osteo-neuro-muscular comorbidities that might interfere in the performance of physical activities in daily life. The study was approved by the Committee for Ethics in Research of both institutions involved, and all subjects gave formal written consent to participate.

\section{Data from Brazil}

In Brazil, data from all subjects were collected at the Laboratory of Research in Respiratory Physiotherapy (Laboratório de Pesquisa em Fisioterapia Pulmonar - LFIP) from the Department of Physiotherapy, Universidade Estadual de Londrina (UEL) and Hospital Universitário Regional 
Norte do Paraná (HURNPR), Londrina. Clinical characteristics of the group of Brazilian patients with COPD are described in table 1.

Londrina is situated in the southern region of Brazil and has approximately 500.000 inhabitants in July 2008. Londrina's only mean of public transport are buses. In the Brazilian group, 30 patients $(75 \%)$ depended on public transport (bus) as transportation mean, whereas 7 patients (17.5\%) used a privately owned car and 3 patients (7.5\%) used ambulance service as transportation mean.

No Brazilian patient was living in a nursing home, and therefore all 40 of them were living in their own homes: 34 patients (85\%) were living with spouse or relatives, and $6(15 \%)$ were living alone.

During the data collection period (throughout the whole year of 2006), Londrina's average temperature in the 4 seasons was: Winter $17.3^{\circ} \mathrm{C}$; Spring $21.1^{\circ} \mathrm{C}$; Summer $24^{\circ} \mathrm{C}$; Autumn $21.5^{\circ} \mathrm{C}$; precipitation was approximately 117 $\mathrm{mm} / \mathrm{month}$; and average humidity was $78 \%{ }^{(12 ; 13)}$. Eight patients $(20 \%)$ were assessed during summer season; 8 (20\%) were assessed during Autumn; most of the patients $(13$, or $32.5 \%)$ were assessed during Winter, whereas 11 patients $(27.5 \%)$ were assessed during Spring.

Concerning education level, 9 patients (22.5\%) were illiterate, whereas 25 patients $(62.5 \%)$ were literate but did not complete primary level education, 2 patients $(5 \%)$ had complete primary level, 2 patients $(5 \%)$ had complete secondary level and 2 patients $(5 \%)$ had complete university level. Regarding race, 26 patients (65\%) were Caucasian, whereas $4(10 \%)$ were black, $8(20 \%)$ were mixed black and white and $2(5 \%)$ had Asian (Japanese) ethnicity. As of 2006, monthly average income for retired individuals in Londrina, Brazil 
corresponded to $355 €^{(14)}$ (or $\mathrm{R} \$ 919$ in Brazilian currency). Clinical characteristics of the group of Brazilian patients with COPD are described in table 1.

\section{Data from Austria}

In Austria, data were collected at the Department of Respiratory and Critical Care Medicine, Otto-Wagner Hospital, Vienna. Vienna is situated in the eastern region of Austria and has approximately 1.675 .000 inhabitants in July 2008. Clinical characteristics of the group of Austrian patients with COPD are described in table 1.

Despite the fact that Vienna has a well developed public transport system (bus, tram, underground and train), less Austrian patients (21, or 52.5\%) depended on the public transport system in comparison to the Brazilian group. More patients $(14$, or $35 \%)$ used a privately owned car. No Austrian patient used ambulance service, but two patients (5\%) declared to use the bicycle as transportation mean. In 3 patients, it was not possible to retrieve information concerning transportation mean.

No Austrian patient was living in a nursing home (all living in their own homes). Twenty-one patients $(52,5 \%)$ were living with spouse or relatives, and $19(47.5 \%)$ were living alone.

During the data collection period (mostly Spring and Summer of 2006), Vienna's average temperature was: Spring $13^{\circ} \mathrm{C}$; Summer $21.5^{\circ} \mathrm{C}$; precipitation was approximately $88 \mathrm{~mm} / \mathrm{month}$; and average humidity was $77 \%{ }^{(15)}$. The majority of patients (25, or $62.5 \% \%$ ) was assessed during Summer, whereas 11 
(or $27.5 \%$ ) were assessed during Spring and only 4 (10\%) were assessed during Autumn or Winter.

Concerning education level, no Austrian patient was illiterate; 29 patients $(72.5 \%)$ had complete primary level education, whereas 6 patients $(15 \%)$ had complete secondary level and 5 patients (12.5\%) had complete university level. All Austrian patients were Caucasian. As of 2006, monthly average income for retired individuals in Vienna, Austria was $1286 €^{(16)}$. Clinical characteristics of the group of Austrian patients with COPD are described in table 1.

\section{Methods}

The objective quantification of physical activities in daily life was performed in both centers with an accelerometer-based activity monitor

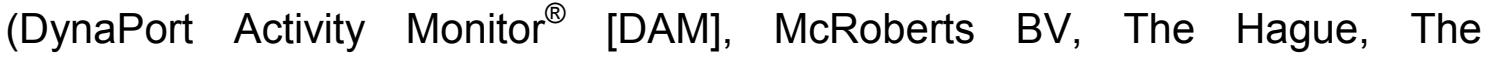
Netherlands). It consists of a small and lightweight box enclosed in a belt worn around the waist and a leg sensor (total weight $=375$ grams). The DAM was recently validated in patients with COPD by being shown to measure the time spent in walking, cycling, standing, sitting or lying as accurately as video recordings ${ }^{(17)}$. Technical specifications of the DAM can be found elsewhere ${ }^{(17)}$. Assessments were done on 2 consecutive week days for 12 hours per day (from waking up time until 12 hours after that) and the average of the 2 days was used for the analysis, since it was shown that this number of days provides an acceptable intraclass correlation coefficient in this population ${ }^{(2)}$. Patients were aware of the assessments aim since this has been shown not to influence the results of activity monitoring in patients with COPD ${ }^{(18)}$. Both in the Austrian 
and Brazilian COPD groups, no assessments were done in days in which the patient performed any informal professional activity.

In the Brazilian center, spirometric assessment was performed using the Pony Cosmed ${ }^{\circledR}$ spirometer (Cosmed, Italy). In the Austrian center, it was performed using the Spirometry PFT unit SensorMedics Vmax 22 (Viasys Healthcare, Germany). The technique in both centers followed the guidelines of the American Thoracic Society/European Respiratory Society ${ }^{(19)}$, and the reference values were those from Quanjer et al. ${ }^{(20)}$.

All patients with COPD reported the degree in which dyspnea influences their activities of daily living through the MMRC scale ${ }^{(21)}$. The 6MWT was performed in both centers according to the guidelines of the American Thoracic Society ${ }^{(22)}$. Two tests were performed with an interval of at least 30 minutes, and the highest 6MWT was used for the analysis. Reference values were those from Troosters et al. ${ }^{(23)}$. Results concerning BMI, FEV 1, MMRC and 6MWT were used to calculate the BODE index as described by Celli et al. ${ }^{(24)}$.

\section{Comorbidities}

In the group of Brazilian patients with COPD, the number (and proportion) of subjects with clinically diagnosed comorbidities was: 15 patients (37.5\%) with systemic hypertension; 13 (32.5\%) with stable cardiac disease; 13 $(32.5 \%)$ with diabetes mellitus and $5(12.5 \%)$ with osteoporosis, besides 9 obese patients $(22.5 \%)$. In the group of Austrian patients with COPD, the number (and proportion) of subjects with clinically diagnosed comorbidities was: 10 patients $(25 \%)$ with systemic hypertension; $15(37.5 \%)$ with stable cardiac disease; 5 (12.5\%) with diabetes melitus and $3(7.5 \%)$ with osteoporosis, 
besides 8 obese patients (20\%). Criteria for considering patients as having systemic hypertension was the current need for antihypertensive medication; for stable cardiac disease was the presence of medical diagnosis of mild atrial arrhythmia treated with medication, ischemic heart disease in the past or dilated cardiomyopathy without significant cardiac functional impairment; for diabetes mellitus and osteoporosis was the patient's self-report in addition to the presence of medical diagnosis of these conditions; and for obesity was BMI>30 $\mathrm{kg} / \mathrm{m}^{2}$.

\section{Statistical Analysis}

Statistical analysis was performed using the GraphPad Prism 3 (GraphPad Software, San Diego, USA). Normal distribution was checked with the Kolmogorov-Smirnov test. Results were described as mean \pm standard deviation. The unpaired $t$ test was used to compare Brazilian patients with COPD and Austrian patients with COPD. Level of significance was set at $p<0.05$ for all the analyses.

\section{RESULTS}

Table 1 shows that Brazilian and Austrian patients with COPD had similar age, BMI, lung function (and therefore GOLD stage), 6MWT, MMRC and BODE index (all $p>0.05$ ). Figure 1 shows that Austrian patients had significantly lower time spent walking ( $40 \pm 36 \mathrm{~min} /$ day versus $56 \pm 32 \mathrm{~min} /$ day; $\mathrm{p}=0.04)$, lower movement intensity $\left(1.5 \pm 0.4 \mathrm{~m} / \mathrm{s}^{2}\right.$ versus $\left.1.9 \pm 0.4 \mathrm{~m} / \mathrm{s}^{2} ; p=0.0001\right)$ and higher time spent sitting $(388 \pm 208 \mathrm{~min} /$ day versus $296 \pm 109 \mathrm{~min} /$ day; $\mathrm{p}=0.02)$ in comparison to the Brazilian patients. In addition, Austrian patients also had 
lower time spent standing (192 $\pm 182 \mathrm{~min} /$ day versus $246 \pm 122 \mathrm{~min} /$ day $)$ and lower time spent lying (98 $\pm 116 \mathrm{~min} /$ day versus $119 \pm 110 \mathrm{~min} / \mathrm{day})$, although these differences did not reach statistical significance $(p=0.12$ and 0.40 , respectively).

Brazilian patients with COPD were divided in two groups based on the involvement $(n=14)$ or not involvement $(n=26)$ in informal professional activities. These two groups did not show significant differences concerning time spent walking $(p=0.76)$, BODE index $(p=0.54)$ and $\mathrm{FEV}_{1}$ (both in absolute values $[p=0.62]$ and percentage of the predicted values $[p=0.56])$. Similarly, dividing the Austrian patients with COPD in two groups involved $(n=5)$ or not involved $(n=35)$ in informal professional activities, there were no differences concerning time spent walking $(p=0.57)$, BODE index $(p=0.34)$ and $\mathrm{FEV}_{1}$ (both in absolute values $[p=0.97]$ and percentage of the predicted values $[p=0.27])$.

In the Austrian group, 19 out of the 40 patients (48\%) did not reach an average of at least 30 minutes of time spent walking per day, which is the minimum amount of physical activity recommended by the American College of Sports Medicine (ACSM) ${ }^{(25)}$. In the Brazilian group, 9 out of the 40 patients $(23 \%)$ did not reach this minimum level of physical activity.

\section{DISCUSSION}

To the authors' best knowledge, this is the first study to compare the level of physical activity in daily life assessed by an advanced and validated activity monitor in patients with COPD from such different origins as Europe and South America, or more specifically Austria and Brazil. The differences could be easily noticed in the present study: firstly, the educational level and economic 
condition of Austrian patients is considerably higher, as one would expect when comparing a developed country with another one in development. Secondly, Brazil is a miscegenated country from the ethnic point of view, in contrast to a typically Caucasian population in Austria. However, despite the fact that lower physical activity level in healthy elderly and adult populations has been associated with non-Caucasian race ${ }^{(6 ; 7)}$, lower educational level ${ }^{(8 ; 9)}$ and lower income $^{(9 ; 10)}$, Austrian patients with COPD showed a significantly lower time spent walking, higher time spent sitting and lower movement intensity in daily life in comparison to matched Brazilian patients with COPD. In addition, the lower physical activity level in daily life in Austrian patients occurred despite the fact that Brazilian patients had a higher prevalence of comorbidities such as hypertension, diabetes and osteoporosis. These results suggest that, in an inactive population such as patients with COPD, the socio-economic and ethnic predictive factors for physical inactivity possibly play a different role than observed in healthy subjects (i.e., worse socio-economic conditions and ethnic miscegenation leads South American patients to be more active than CentralEuropean patients, in opposition to what would be expected from previous studies in healthy subjects). These results can be confirmed by the fact that the time spent walking in daily life in the Brazilian COPD group ( $56 \pm 32$ minutes per day) was also markedly higher than the observed in a previous sample from Belgium $\left.^{(2)}\right](44 \pm 26$ minutes per day), which by the way was very similar to the

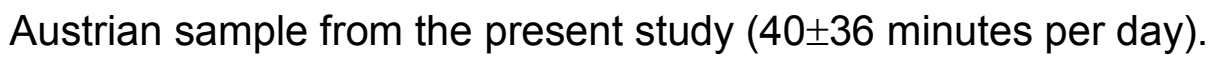

The differences in daily physical activity level between Austrian and Brazilian patients with COPD cannot be explained by anthropometrical and clinical differences between the groups. The groups were matched for age, 
gender, BMI, lung function, functional exercise capacity, disease severity as measured both by the GOLD stages and the BODE index, and even matched for the presence of cardiac disease and obesity as comorbidities. Furthermore, in patients with COPD, the use of LTOT may further reduce daily physical activity level ${ }^{(18)}$, but this did not influence the present results since no patient in both groups was using LTOT. These factors seem to point out that, in patients with COPD, socio-economic and ethnic factors are responsible for impacting differently on daily physical activity as one would expect from studies with healthy subjects. A study performed with a sample of Spanish patients with COPD has also shown that lower physical activity level was related to higher socioeconomic status ${ }^{(26)}$, confirming the present results. The socio-economic factors which might lead patients in Brazil to be more active than patients in Austria include the fact that the proportion of Brazilian patients who have access to a privately owned transportation mean was half of the Austrian patients, and therefore Brazilian patients have to walk more and use more the public transport, in addition to the fact that Brazilian patients have a lower degree of support by the healthcare system. Since $85 \%$ of Brazilian patients were living with spouse or relatives (in comparison to $52.5 \%$ in the Austrian group), it may also be argued that this can be a motivating factor in order to undertake more outdoor social activities with the family. Ethnic differences are likely linked to the socio-economic discrepancies between the groups.

A study by $\mathrm{He}$ et al. ${ }^{(27)}$ has showed that leisure time physical activity in healthy adults with 51-61 years of age was higher in Caucasian than in nonCaucasian subjects. On the other hand, work-related physical activity showed the reverse pattern, being highest in non-Caucasian subjects with lower 
education. These variations in physical activity level related to work or leisure cannot explain the present results, since all patients in both groups were officially retired, the vast majority of them were in fact no longer involved in any professional activity and those still involved in informal professional activities were not more active than those not involved. Therefore, in spite of the fact that more Brazilian patients were involved in informal professional activities, this did not influence the results since daily physical activity assessments in the present study were not performed in days in which patients performed any professional activity. In case assessments were performed in days in which the patients were involved in informal working, the differences in daily physical activity level between the Brazilian and Austrian groups would possibly have been even larger. On the other hand, it cannot be ruled out that the professional activity performed by these individuals before retirement might have influenced their current physical activity habits.

Environmental factors are also known to influence daily physical activity level. However, data collection in the present study was performed during a period in which mean temperatures, humidity and precipitation were similar between both countries (the vast majority of patients in both groups was assessed in periods when mean temperatures were quite similar). In addition, two other factors suggest that environmental factors did not play a role in the present results: Firstly, the city of Londrina is located in the southern region of Brazil, i.e., the less warm region in the country and with more resemblance to the European general climate. Secondly, Togo et al. ${ }^{(28)}$ observed that, in healthy elderly, the amount of steps per day increased with the mean ambient temperature over the range of $-2^{\circ} \mathrm{C}$ to $17^{\circ} \mathrm{C}$, but decreased over the range of 
$17^{\circ} \mathrm{C}$ to $29^{\circ} \mathrm{C}$. Therefore, in case weather was an influencing factor in the present results, activity level would supposedly be lower in Brazilian patients than in Austrian patients.

Another interesting finding from the present study was that the proportion of Brazilian patients with COPD who reached the minimum amount of daily physical activity recommended by the ACSM ${ }^{(25)}$ was more than twice the proportion of Austrian patients. These results directly affect treatment options, since they indicate that pulmonary rehabilitation programs in Central Europe, even more than in South America, should prioritize the goal of enhancing daily physical activity. Furthermore, since non-compliance with these recommendations is related to higher risk of death in retired elderly ${ }^{(29 ; 30)}$, early mortality would be expected to be higher in Austrian patients than in Brazilian patients, although ethnicity and annual income were not predictive factors of mortality in a sample of American patients with severe emphysema ${ }^{(31)}$. This topic surely deserves deeper investigation.

In summary, Austrian patients with COPD showed a significantly lower daily physical activity level in comparison to matched Brazilian patients with COPD. Socio-economic and ethnic factors appear to influence stable patients with COPD differently than described in previous studies for healthy elderly and adults.

\section{ACKNOWLEDGMENTS AND FUNDING}

The authors would like to thank all professionals involved in the data collection in both centers. The Brazilian part of the study was funded by a grant from the National Council for Scientific and Technological Development (CNPq, 
Brazil). FP and AFB are grant-holders as researchers of the CNPq. MKB was funded by the Ludwig Boltzmann Institute for COPD, Vienna, Austria.

\section{Conflict of interest statement}

Fabio Pitta states that no financial or other potential conflicts of interest exist. Marie-Kathrin Breyer states that no financial or other potential conflicts of interest exist. Nidia A. Hernandes states that no financial or other potential conflicts of interest exist. Denilson Teixeira states that no financial or other potential conflicts of interest exist. Thaís J. P. Sant'Anna states that no financial or other potential conflicts of interest exist.

Andréa D. Fontana states that no financial or other potential conflicts of interest exist. Vanessa S. Probst states that no financial or other potential conflicts of interest exist. Antonio F. Brunetto states that no financial or other potential conflicts of interest exist. Martijn A. Spruit states that no financial or other potential conflicts of interest exist.

Emiel F. M. Wouters states that no financial or other potential conflicts of interest exist. Otto C. Burghuber states that no financial or other potential conflicts of interest exist. Sylvia Hartl states that no financial or other potential conflicts of interest exist. 


\section{REFERENCES}

1. Pitta F, Troosters T, Probst VS, Spruit MA, Decramer M, Gosselink R. Quantifying physical activity in daily life with questionnaires and motion sensors in COPD. Eur Respir J 2006 May;27(5):1040-55.

2. Pitta F, Troosters T, Spruit MA, Probst VS, Decramer M, Gosselink R. Characteristics of physical activities in daily life in chronic obstructive pulmonary disease. Am J Respir Crit Care Med 2005 May 1;171(9):9727.

3. Singh S, Morgan MD. Activity monitors can detect brisk walking in patients with chronic obstructive pulmonary disease. J Cardiopulm Rehabil 2001 May;21(3):143-8.

4. Schonhofer B, Ardes P, Geibel M, Kohler D, Jones PW. Evaluation of a movement detector to measure daily activity in patients with chronic lung disease. Eur Respir J 1997 Dec;10(12):2814-9.

5. Coronado M, Janssens JP, de Muralt B, Terrier P, Schutz Y, Fitting JW. Walking activity measured by accelerometry during respiratory rehabilitation. J Cardiopulm Rehabil 2003 Sep;23(5):357-64.

6. Marshall SJ, Jones DA, Ainsworth BE, Reis JP, Levy SS, Macera CA. Race/ethnicity, social class, and leisure-time physical inactivity. Med Sci Sports Exerc 2007 Jan;39(1):44-51. 
7. Ransdell LB, Wells CL. Physical activity in urban white, AfricanAmerican, and Mexican-American women. Med Sci Sports Exerc 1998 Nov;30(11):1608-15.

8. Martinez-Gonzalez MA, Varo JJ, Santos JL, De Irala J, Gibney M, Kearney $\mathrm{J}$, et al. Prevalence of physical activity during leisure time in the European Union. Med Sci Sports Exerc 2001 Jul;33(7):1142-6.

9. Crespo CJ, Ainsworth BE, Keteyian SJ, Heath GW, Smit E. Prevalence of physical inactivity and its relation to social class in U.S. adults: results from the Third National Health and Nutrition Examination Survey, 19881994. Med Sci Sports Exerc 1999 Dec;31(12):1821-7.

10. Parks SE, Housemann RA, Brownson RC. Differential correlates of physical activity in urban and rural adults of various socioeconomic backgrounds in the United States. J Epidemiol Community Health 2003 Jan;57(1):29-35.

11. Rabe KF, Hurd S, Anzueto A, Barnes PJ, Buist SA, Calverley P, et al. Global strategy for the diagnosis, management, and prevention of chronic obstructive pulmonary disease: GOLD executive summary. Am J Respir Crit Care Med 2007 Sep 15;176(6):532-55.

12. www.inmet.gov.br. Last accessed: July 2008.

13. www iapar br . Last accessed: July 2008.

14. www.diariopopularpr.com.br . Last accessed: July 2008.

15. www.zagm.ac.at . Last accessed: July 2008. 
16. www.statistik.at . Last accessed: July 2008.

17. Pitta F, Troosters T, Spruit MA, Decramer M, Gosselink R. Activity Monitoring for Assessment of Physical Activities in Daily Life in Patients With Chronic Obstructive Pulmonary Disease. Arch Phys Med Rehabil 2005 Oct;86(10):1979-85.

18. Sandland CJ, Singh SJ, Curcio A, Jones PM, Morgan MD. A profile of daily activity in chronic obstructive pulmonary disease. J Cardiopulm Rehabil 2005 May;25(3):181-3.

19. Miller MR, Hankinson J, Brusasco V, Burgos F, Casaburi R, Coates A, et al. Standardisation of spirometry. Eur Respir J 2005 Aug;26(2):319-38.

20. Quanjer PH, Tammeling GJ, Cotes JE, Pedersen OF, Peslin R, Yernault JC. Lung volumes and forced ventilatory flows. Report Working Party Standardization of Lung Function Tests, European Community for Steel and Coal. Official Statement of the European Respiratory Society. Eur Respir J Suppl 1993 Mar;16:5-40.

21. Mahler DA, Wells CK. Evaluation of clinical methods for rating dyspnea. Chest 1988 Mar;93(3):580-6.

22. ATS statement: guidelines for the six-minute walk test. Am J Respir Crit Care Med 2002 Jul 1;166(1):111-7.

23. Troosters T, Gosselink R, Decramer M. Six minute walking distance in healthy elderly subjects. Eur Respir J 1999 Aug;14(2):270-4. 
24. Celli BR, Cote CG, Marin JM, Casanova C, Montes dO, Mendez RA, et al. The body-mass index, airflow obstruction, dyspnea, and exercise capacity index in chronic obstructive pulmonary disease. N Engl J Med 2004 Mar 4;350(10):1005-12.

25. Pate RR, Pratt M, Blair SN, Haskell WL, Macera CA, Bouchard C, et al. Physical activity and public health. A recommendation from the Centers for Disease Control and Prevention and the American College of Sports Medicine. JAMA 1995 Feb 1;273(5):402-7.

26. Garcia-Aymerich J, Felez MA, Escarrabill J, Marrades RM, Morera J, Elosua R, et al. Physical activity and its determinants in severe chronic obstructive pulmonary disease. Med Sci Sports Exerc 2004 Oct;36(10):1667-73.

27. He XZ, Baker DW. Differences in leisure-time, household, and workrelated physical activity by race, ethnicity, and education. J Gen Intern Med 2005 Mar;20(3):259-66.

28. Togo F, Watanabe E, Park H, Shephard RJ, Aoyagi Y. Meteorology and the physical activity of the elderly: the Nakanojo Study. Int J Biometeorol 2005 Nov;50(2):83-9.

29. Hakim AA, Petrovitch H, Burchfiel CM, Ross GW, Rodriguez BL, White LR, et al. Effects of walking on mortality among nonsmoking retired men. N Engl J Med 1998 Jan 8;338(2):94-9. 
30. LaCroix AZ, Leveille SG, Hecht JA, Grothaus LC, Wagner EH. Does walking decrease the risk of cardiovascular disease hospitalizations and death in older adults? J Am Geriatr Soc 1996 Feb;44(2):113-20.

31. Martinez FJ, Foster G, Curtis JL, Criner G, Weinmann G, Fishman A, et al. Predictors of mortality in patients with emphysema and severe airflow obstruction. Am J Respir Crit Care Med 2006 Jun 15;173(12):1326-34. 
Table 1. Characteristics of the groups of patients with COPD from Austria and Brazil.

\begin{tabular}{lcc}
\hline & COPD Austria & COPD Brazil \\
& $(\mathrm{n}=40)$ & $(\mathrm{n}=40)$ \\
\hline Age (years) & $63 \pm 7$ & $66 \pm 8$ \\
Gender (M / F) & $21 / 19$ & $18 / 22$ \\
Smoking (pack/years) & $44 \pm 13$ & $40 \pm 34$ \\
BMI (kg/m ${ }^{2}$ ) & $26 \pm 4$ & $26 \pm 6$ \\
GOLD (II / III / IV) & $17 / 14 / 9$ & $16 / 18 / 6$ \\
FEV (\%predicted) & $48 \pm 17$ & $46 \pm 17$ \\
FVC (\%predicted) & $78 \pm 17$ & $75 \pm 22$ \\
MMRC (0 to 4) & $2.8 \pm 1.1$ & $2.4 \pm 1.1$ \\
6MWT (\%predicted) & $68 \pm 19$ & $71 \pm 19$ \\
BODE index (0 to 10) & $4.0 \pm 2.6$ & $4.4 \pm 2.1$ \\
\hline
\end{tabular}

Data are shown as median \pm standard deviation (except for gender and GOLD stages). Comparison between patients with COPD from Austria and Brazil was performed with the unpaired t test except for MMRC and BODE index, which were analyzed with the Mann-Whitney test. There were no statistically significant differences between the groups. COPD = Chronic Obstructive Pulmonary Disease; $\mathrm{M}=$ male; $\mathrm{F}=$ female; $\mathrm{BMI}=$ body mass index; $\mathrm{kg} / \mathrm{m}^{2}=$ kilograms per meters squared; GOLD = Global Initiative for Obstructive Lung Disease (stages II, III and IV). $\mathrm{FEV}_{1}=$ Forced Expiratory Volume in the First Second; FVC = forced vital capacity; MMRC = modified version of the Medical Research Council scale; 6MWT = 6-minute walking test. 
Figure 1.

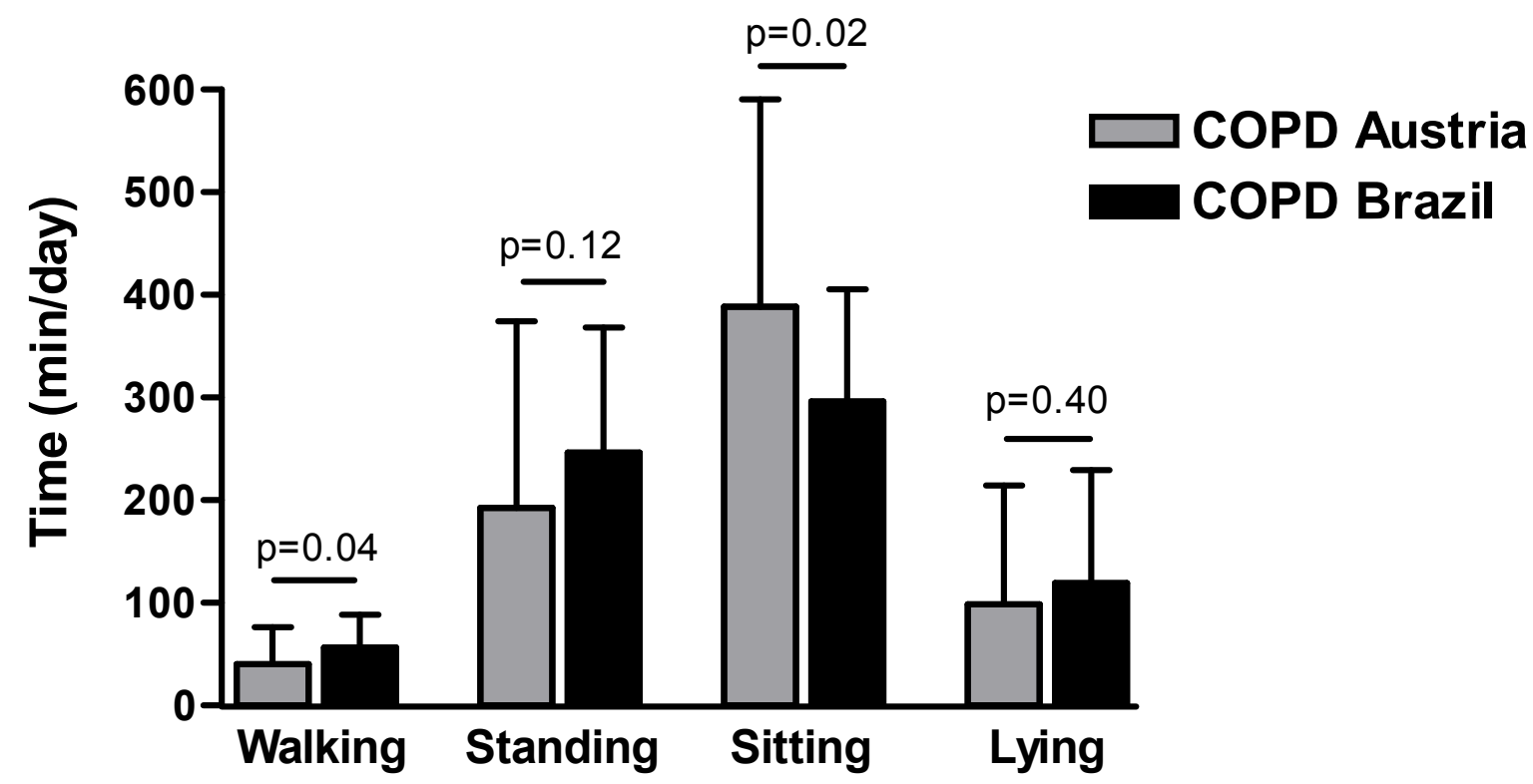

Figure 1. Comparison of time spent walking, standing, sitting and lying per day between Austrian patients with COPD $(n=40)$ and Brazilian patients with COPD $(n=40)$. Statistical analysis was performed with the unpaired t test. 


\section{CONCLUSÕES}




\section{CONCLUSÕES}

Considerando os resultados de ambos os estudos apresentados nesta dissertação pode-se concluir que pacientes portadores de DPOC brasileiros são menos ativos em suas atividades físicas de vida diária quando comparados a idosos saudáveis, além de caminharem com menor intensidade de movimento. Além disso, o tempo gasto andando na vida diária desses pacientes correlacionou-se apenas moderadamente com a capacidade funcional e máxima de exercício. No entanto, quando grupos pareados de pacientes do Brasil e da Áustria foram comparados, os pacientes brasileiros demonstraram-se significativamente mais ativos fisicamente em sua vida diária do que os pacientes austríacos. 


\section{REFERÊNCIAS BIBLIOGRÁFICAS}

1. Rabe KF, Hurd S, Anzueto A, Barnes PJ, Buist SA, Calverley P, et al. Global strategy for the diagnosis, management, and prevention of chronic obstructive pulmonary disease: GOLD executive summary. Am J Respir Crit Care Med 2007;176(6):532-55.

2. Casaburi R, ZuWallack R. Pulmonary rehabilitation for management of chronic obstructive pulmonary disease. N Engl J Med 2009;360(13):132935 .

3. Troosters T, Casaburi R, Gosselink R, Decramer M. Pulmonary rehabilitation in chronic obstructive pulmonary disease. Am J Respir Crit Care Med 2005;172(1):19-38.

4. Barnes PJ, Celli BR. Systemic manifestations and comorbidities of COPD. Eur Respir J 2009;33(5):1165-85.

5. Lacasse Y, Brosseau L, Milne S, Martin S, Wong E, Guyatt GH, et al. Pulmonary rehabilitation for chronic obstructive pulmonary disease. Cochrane Database Syst Rev 2002;(3):CD003793.

6. Yohannes AM, Baldwin RC, Connolly M. Mortality predictors in disabling chronic obstructive pulmonary disease in old age. Age Ageing 2002;31(2):137-40.

7. Garcia-Aymerich J, Lange $P$, Benet $M$, Schnohr $P$, Anto JM. Regular physical activity reduces hospital admission and mortality in chronic obstructive pulmonary disease: a population based cohort study. Thorax 2006;61(9):772-8.

8. Pitta F, Troosters T, Probst VS, Spruit MA, Decramer M, Gosselink R. Quantifying physical activity in daily life with questionnaires and motion sensors in COPD. Eur Respir J 2006;27(5):1040-55.

9. Benzo R. Activity monitoring in chronic obstructive pulmonary disease. J Cardiopulm Rehabil Prev 2009;29(6):341-7.

10. Pate RR, Pratt M, Blair SN, Haskell WL, Macera CA, Bouchard C, et al. Physical activity and public health. A recommendation from the Centers for Disease Control and Prevention and the American College of Sports Medicine. JAMA 1995;273(5):402-7.

11. Pitta F, Troosters T, Spruit MA, Probst VS, Decramer M, Gosselink R. Characteristics of physical activities in daily life in chronic obstructive pulmonary disease. Am J Respir Crit Care Med 2005;171(9):972-7.

12. Pitta F, Troosters T, Probst VS, Lucas S, Decramer M, Gosselink R. Potential consequences for stable chronic obstructive pulmonary disease 
patients who do not get the recommended minimum daily amount of physical activity. J Bras Pneumol 2006;32(4):301-8.

13. Pitta F, Troosters T, Probst VS, Spruit MA, Decramer M, Gosselink R. Physical activity and hospitalization for exacerbation of COPD. Chest 2006;129(3):536-44.

14. Pitta F, Troosters T, Probst VS, Langer D, Decramer M, Gosselink R. Are patients with COPD more active after pulmonary rehabilitation? Chest 2008;134(2):273-80.

15. Schonhofer B, Ardes P, Geibel M, Kohler D, Jones PW. Evaluation of a movement detector to measure daily activity in patients with chronic lung disease. Eur Respir J 1997;10(12):2814-9.

16. Watz H, Waschki B, Meyer T, Magnussen H. Physical activity in patients with chronic obstructive pulmonary disease. Eur Respir J 2009;33:262-72.

17. Marshall SJ, Jones DA, Ainsworth BE, Reis JP, Levy SS, Macera CA. Race/ethnicity, social class, and leisure-time physical inactivity. Med Sci Sports Exerc 2007;39(1):44-51.

18. Parks SE, Housemann RA, Brownson RC. Differential correlates of physical activity in urban and rural adults of various socioeconomic backgrounds in the United States. J Epidemiol Community Health 2003;57(1):29-35.

19. Ransdell LB, Wells CL. Physical activity in urban white, African-American, and Mexican-American women. Med Sci Sports Exerc 1998;30(11):1608-15.

20. Martinez-Gonzalez MA, Varo JJ, Santos JL, de IJ, Gibney M, Kearney J, et al. Prevalence of physical activity during leisure time in the European Union. Med Sci Sports Exerc 2001;33(7):1142-6. 
ANEXOS 


\section{Anexos}

\section{Anexo 1 - Normas para publicação no periódico Jornal Brasileiro de Pneumologia (ISSN-1806-3713)}

\section{APRESENTAÇÃO E SUBMISSÃO DOS MANUSCRITOS}

Os manuscritos deverão ser obrigatoriamente encaminhados via eletrônica a partir da própria home-page do Jornal.

Com exceção das unidades de medidas, siglas e abreviaturas devem ser evitadas ao máximo, devendo ser utilizadas apenas para termos consagrados. Estes termos estão definidos na Lista de Abreviaturas e Acrônimos aceitos sem definição. Quanto a outras abreviaturas, sempre definilas na primeira vez em que forem citadas, por exemplo: proteína $C$ reativa (PCR). Após a definição da abreviatura, o termo completo não deverá ser mais utilizado. Com exceção das abreviaturas aceitas sem definição, elas não devem ser utilizadas nos títulos e evitadas no resumo dos manuscritos se possível. Ao longo do texto igualmente evitar a menção ao nome de autores, dando-se sempre preferência às citações numéricas apenas.

Quando os autores mencionarem qualquer substância ou equipamento incomum, deverão incluir o modelo/número do catálogo, o nome da fabricante, a cidade e o país, por exemplo: "... esteira ergométrica (modelo ESD-01; FUNBEC, São Paulo, Brasil)...". No caso de produtos provenientes dos EUA e Canadá, o nome do estado ou 
província também deverá ser citado; por exemplo: "... tTG de fígado de porco da Guiné (T5398; Sigma, St. Louis, MO, EUA) ..."

\section{PREPARO DO MANUSCRITO}

Com exceção de trabalhos considerados de excepcional complexidade, a revista considera 6 o número máximo aceitável de autores. No caso de maior número de autores, enviar carta à Secretaria do Jornal descrevendo a participação de cada um no trabalho.

A página de identificação deve conter o título do trabalho, em português e inglês, nome completo e titulação dos autores, instituições a que pertencem, endereço completo, inclusive telefone, fax e e-mail do autor principal, e nome do órgão financiador da pesquisa, se houver.

Resumo: deve conter informações facilmente compreendidas, sem necessidade de se recorrer ao texto, não excedendo 250 palavras. Deve ser feito na forma estruturada com: Objetivo, Métodos, Resultados e Conclusões. Quando se tratar de artigos de Revisão e Relatos de Casos o Resumo não deve ser estruturado. Para Comunicações Breves não deve ser estruturado nem exceder 100 palavras.

Abstract: uma versão em língua inglesa, correspondente ao conteúdo do Resumo deve ser fornecida.

Descritores e Keywords: Deve ser fornecido de três a seis termos em português e inglês, que definam o assunto do trabalho. Devem ser, obrigatoriamente, baseados nos DeCS (Descritores em Ciências da Saúde), publicados pela Bireme e disponíveis no endereço eletrônico: 
http://decs.bvs.br, enquanto os keywords em inglês devem ser baseados nos MeSH (Medical Subject Headings) da National Library of Medicine, disponíveis no endereço eletrônico www.nlm.nih.gov/mesh/MBrowser.html.

Texto:

Artigos originais: o texto deve ter entre 2000 e 3000 palavras, excluindo referências e tabelas. Deve conter no máximo 5 tabelas e/ou figuras. O número de referências bibliográficas não deve exceder 30 . A sua estrutura deve conter as seguintes partes: Introdução, Métodos, Resultados, Discussão, Agradecimentos e Referências. A seção Métodos deverá conter menção a aprovação do estudo pelo Comitê de Ética em Pesquisa em Seres Humanos, ou pelo Comitê de Ética em Pesquisa em Animais, ligados a Instituição onde o projeto foi desenvolvido. Nessa seção também deve haver descrição da análise estatística empregada, com as respectivas referências bibliográficas. Ainda que a inclusão de subtítulos no manuscrito seja aceitável, o seu uso não deve ser excessivo e deve ficar limitado às sessões Métodos e Resultados somente.

Tabelas e Figuras: tabelas e gráficos devem ser apresentados em preto e branco, com legendas e respectivas numerações impressas ao pé de cada ilustração. As tabelas e figuras devem ser enviadas no seu arquivo digital original, as tabelas preferencialmente em arquivos Microsoft Word e as figuras em arquivos Microsoft Excel, Tiff ou JPG. Fotografias de exames, procedimentos cirúrgicos e biópsias onde foram utilizadas colorações e técnicas especiais serão consideradas para impressão colorida, sem custo adicional aos autores. As grandezas, unidades e símbolos devem obedecer às normas nacionais correspondentes (ABNT: http://www.abnt.org.br). 
Legendas: legendas deverão acompanhar as respectivas figuras (gráficos, fotografias e ilustrações) e tabelas. Cada legenda deve ser numerada em algarismos arábicos, correspondendo a suas citações no texto. Além disso, todas as abreviaturas e siglas empregadas nas figuras e tabelas devem ser definidas por extenso abaixo das mesmas.

Referências: devem ser indicadas apenas as referências utilizadas no texto, numeradas com algarismos arábicos e na ordem em que foram citadas. A apresentação deve estar baseada no formato Vancouver Style, atualizado em outubro de 2004. Os títulos dos periódicos citados devem ser abreviados de acordo com o estilo apresentado pela List of Journal Indexed in Index Medicus, da National Library of Medicine. Para todas as referências, cite todos os autores até seis. Acima desse número, cite os seis primeiros autores seguidos da expressão et al. 
Anexo 2 - Normas para publicação no periódico Respiratory Medicine (ISSN-0954-6111)

FORMATO E ESTRUTURA DO MANUSCRITO

$\mathrm{O}$ arquivo deve estar preferencialmente no formato Microsoft Word. Em geral, os artigos devem apresentar a estrutura convencional contendo: "Summary", "Introduction", "Methods", “Results", "Discussion” e "References".

Título

A página-título deve conter título redigido em letras caixa alta (não excedendo 100 letras), nome dos autores e instituições. O autor e o endereço para correspondência devem ser claramente indicados, incluindo telefone, fax e e-mail do autor.

Abstract

Um "abstract" contendo o conteúdo do manuscrito resumidamente, com no máximo 250 palavras, deve ser redigido em uma página distinta.

Formato das referências

Os manuscritos devem seguir as padronizações propostas pelo Vancouver Style para referências, como a seguir:

Texto: indicar as referências utilizando números arábicos sobrescritos ao longo do texto. O nome do autor pode ser referenciado, porém, o número da referência deve ser sempre citado. 
Lista: o número das referências na lista devem respeitar a ordem na qual aparecem no texto.

Figuras

Figuras de boa qualidade podem ser submetidas online em arquivos separadamente. Permissão para reprodução de figuras deve ser sempre obtida previamente.

Tabelas

As tabelas devem ser submetidas online em arquivos separadamente acompanhada por um título descritivo e numeradas com números arábicos. Tabelas devem ser citadas ao longo do texto.

Palavras-chave

Uma lista contendo até seis palavras-chave deve ser providenciada.

Idioma

Os manuscritos serão aceitos apenas quando forem redigidos na língua inglesa. 